THE SEARCH FOR HEAVY IEPTONS AND MUON-ELECTRON DIFTERENCES*

Martin I. Perl and Petros Rapiais Stanford Tinear Accelerator Center Stanford University, Stanford, California 94305

Revised version of review paper originally presented at the Muon Physics Conference, Colorado State University, September 6-10, 1971

To be published in the Proceedings of the Muon Physics Conference.

Aork supported by the U.S. Atomic Energy Commission

This poper replaces and supersedes SIAC-PUB-982 and SIAC-PUB-1062.
I. Introduction

II. Some Possible Types of Heavy Leptons
A. Heavy Sequential Leptons: $\mu^{\prime}, \mu^{\prime \prime} \ldots$
B. Heevy Excited Leptons: $e^{*}, \mu^{*}$
C. Special Pairs of Heavy Leptons
D. Stable and Very Long-Lived Heavy Leptons
E. Other Possibilities
F. A Word on Notation

III. The Decey Properties of the Heavy Leptons
A. Charged Heavy Sequential Leptons
B. Neutral Heavy Sequential Leptons
c. Special Pairs of Leptons
D. Charged Heavy Excited Leptons

IV. Searches for Low Mass or Stable Heavy Ieptons

A. Searches in the Decay Modes of the Pion and Kaon
1. The Method
2. Past Searches
3. Future Seacches

B. Searches in Particle Beams for Short-Lived Cherged Heavy Leptons
1. The Method
2. Past Searches
3. Future Searches

c. Searches for Stable Charged Heavy Leptons in Particle Beams
1. The Method
2. Tast searches
3. Future Searches 
v. Searches for Unstable Heavy Leptuns with Masses Greater Than About $0.5 \mathrm{GeV} / \mathrm{c}^{2}$

A. Detection Method

B. Electron-Positron Colliding Beams Production of Heavy Leptons
1. The Method
2. Past Searches
3. Present and Future Searches

C. Photoproduction of Heavy Leptons

1. The Method

2. Past Searches

\section{Future Searches}

D. Heavy Lepton Production in Proton-Proton Collisions

F. Searches for Heavy Excited Leptons Using Charged Lepton-Proton Eleastic Scattering
1. The Method
2. Past Searches

3. Future Searches

F. Searches for Heavy Excited Leptons in Lepton Bremsstrahlung
1. The Method
2. Past Searches
3. Future Searches
G. Searches for Heavy Leptons Using Charged Lepton-Proton Inelastic Scattering
H. Searches for Special Pair Heavy Leptons Using Neutrino-Nucleon Inelastic Scattering

VI. Comparison of Some Static Properties of the Mhon and the Electron
A. Electric Charge
B. Gyromagnetic Ratio

VIT. Mu-Mesic Atoms
VIII. High Energy Reactions of Muons and Electrons

IX. Muon-Proton Elastic Scattering and Form Factors

A. Theoretical Background

B. Experimental Results

x. Muon-Proton Inelastic Scattering

A. Theoretical Background

B. Experimental Fesults

XI. Charged Lepton F'orm Factors in Colliding Beam Experiments

A. Elastic Electron-Elcctron Scattering: $e^{-}+e^{-} \rightarrow e^{-}+e^{-} \quad 70$

B. Bhabha Seattering: $e^{-}+e^{+} \rightarrow e^{-}+e^{+} \quad 71$

C. Muon Pair Production: $\mathrm{e}^{-}+\mathrm{e}^{+} \rightarrow \mu^{-}+\mu^{+} \quad 73$

XII. Speculations

A. Searches for Heavy Leptons

B. Charged-Lepton Form Factors

C. Anomalous Lepton-Hadron Interactions

References
6

6 
I. TNTRODUCTION

This paper is a review of some recent experimental studies on the fundamental nature of the mon and the electron, and on the relationship between these particles. The paper begins with a summary of our present knowledge as to the existence of higher mass charged leptons or of nonzero mass neutral leptons. This is followed by a brief discussion of some of the static and atomic properties of the muon and electron. The final portion of the paper is then concerned with the high energy behavior of the charged leptons in elcctromagnctic and strong interaction processes. Only a few references will be made to the behavior of the charged leptons in weak interaction processes.

For this audience there is no need to present an extended description of what we know about the fundamental nature of the muon and the electron. Therefore, we simply present in Table I a summary of our present knowledge -- or better a portrait -- of the muon and the electron. The set of properties (Iisted in Table I) that the muon and electron possess in conmon are collectively described by the phrase "mon-electron universality."

This portrait of the charged leptons leads to numerous questions. Arc there heavier charged leptons? If there are no heavier charged leptons, why are there two charged leptons? Are the charged leptons really point particles, or do they have a structure which has not yet been detected? Are the electron and muon related in any profound way, or are they simply unrelated particles, both of which just happen to obey the Dirac equations? Are there differences between the muon and the electron other than those listed in Table I?

$$
-1-
$$

Table I

\begin{tabular}{|c|c|c|c|}
\hline Property & $\begin{array}{l}\text { Comparison Between } \\
\text { Muon and Electron }\end{array}$ & $\begin{array}{l}\text { If Pron } \\
\text { Muon }\end{array}$ & $\begin{array}{l}\text { is Different } \\
\text { Electron }\end{array}$ \\
\hline Intrinsic spin & both $1 / 2$ & & \\
\hline Statistics & both Fermi-Dirac & & \\
\hline Fundamental equation & both Dirgc equation & & \\
\hline Structure & $\begin{array}{l}\text { both point particles } \\
\text { (within present ex- } \\
\text { perimental precision } \\
\text { as discussed in this } \\
\text { article) }\end{array}$ & & \\
\hline $\begin{array}{l}\text { Interact through the } \\
\text { strong interactions }\end{array}$ & $\begin{array}{l}\text { both no (within present } \\
\text { experimental precision } \\
\text { as discussed in this } \\
\text { article) }\end{array}$ & & \\
\hline $\begin{array}{l}\text { Interact through the electro- } \\
\text { magnetic interaction }\end{array}$ & both yes & & \\
\hline Magnitude or electric charge & same for both & & \\
\hline Sign of electric charge & $\begin{array}{l}\text { both }+ \text { or }- \text {, } \\
\text { neither } 0\end{array}$ & & \\
\hline Gyromagnetic ratio & $\begin{array}{l}\text { both given by quantum } \\
\text { electrodynamics and } \\
\text { particle's mass }\end{array}$ & & \\
\hline $\begin{array}{l}\text { Interact through the weak } \\
\text { interactions }\end{array}$ & both yes & & \\
\hline $\begin{array}{l}\text { Magnitude of weak inter- } \\
\text { action coupling constant }\end{array}$ & same for both & & \\
\hline Associated neutrino & $\begin{array}{l}\text { yes but different } \\
\text { neutrinos }\end{array}$ & $\nu_{\mu}$ & $v_{\mathrm{e}}$ \\
\hline Mass $\left(\mathrm{MeV} / \mathrm{c}^{2}\right)$ & & 106 & 0.51 \\
\hline
\end{tabular}

We must admit that at present we do not possess a fundamental theory which can provide answers to these questions. We must also admit that we do not, even possess a theory which can guide us as to how we might try

$$
-2 \text { - }
$$


to answer these questions experimentally. Therefore the experimenter is on his own in searching for answers to these questions. These searches, which in their very nature mast be speculative, have taken two directions. One direction consists of attempts to find heavier members of the electronmuon family. The other direction consists of comparative measurements of the properties of the muon and of the electron in the hope that hitherto unknown differences between the two particles will be discovered. Of course, for this second direction to be fruitful, one must measure known properties with greater precision or one must measure properties which have not been previously measured. The recent high precision measurements of the grromagnetic ratio of the mon are an illustration of the first type of measurement. ${ }^{1}$ The deep inelastic scattering experiment, which we will describe later, is an illustration of the second type of measurement.

A number of comprehensive reviews of the properties of the muon and the electron have appeared in the last ten years. ${ }^{2,3,4}$ we shall not repeat the material contained in those reviews, but only summarize their conclusions. Thus our emphasis will be on very recent experimental results. These new results have not altered the portrait, presented in Table I, in an experimentally significant way. But these new results do indicate what could be the most fruitful directions for future investigation. This forms the subject of the last section of this paper -. the section entitled "Speculations."
II. SOME POSSTBLE TYPES OF HEAVY IETTONS

To simplify the discussion of past and future searches for heavy leptons, we shall consider four experimental classes of thesc hypothctical particles. The first three classes are defined according to the leptonic properties of the leptons.

A. Heavy Sequential Leptons: $\mu^{\prime}, \mu^{\prime \prime} \ldots$

Suppose heavy leptons exist in the mass sequence

$$
e, \mu, \mu^{\prime}, \mu^{\prime \prime} \cdots \text {, }
$$

with associated neutrinos

$$
v_{e}, \nu_{\mu}, \nu_{\mu}, v_{\mu}, \ldots .
$$

Further suppose that each charged lepton and its associated neutrino possess a unique lepton number property which is different from the lepton number possessed by every other charged lepton-neutrino pair, and that these lepton numbers $\left(n_{\mu^{\prime}}\right)$ are separately conserved in strong, electromagnetic and weak interactions. In particular there are no electromagnetic vertexes of the form shown in Fig. 1;

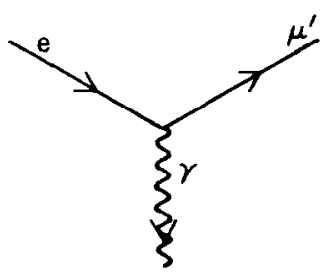

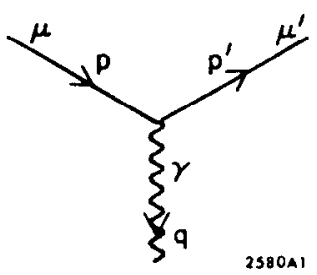

2580A1 
and the charged heavy sequential leptons cannot decay electromagnetically. We refer to the charged leptons or their associated neutrinos as heavy sequential leptons and use the respective symbols $\mu^{\prime}$ and $\nu_{\mu^{\prime}}$. The use of the term sequential emphasizes that the properties of these heavy leptons follow in the main sequence of the $e$ and the $\mu$. In particular, unless we state otherwisc, we assume that the mass of the associated neutrino is zero.

And following the e and $\mu$ conventions, the lepton number $n_{\mu^{\prime}}=+1$ is associated with the $\mu^{\prime-}$ and $\nu_{\mu^{\prime}}$. In writing reactions we shall generally use the $\mu^{\prime}$ as an example and for brevity omit the corresponding $\mu^{\prime+}$ reaction.

\section{B. Heavy Excited Ieptons: $e^{*}, \mu^{*}$}

Suppose there exists a heavy charged lepton which possesses the same lepton number property as the electron, or the mon, of the same sign of inarge. Then the electromagnetic couplings just pictured are allowed, and tne electromagnetic decays

$$
e^{ \pm \pm} \rightarrow e^{ \pm}+\gamma, \quad \mu * \pm+\mu^{ \pm}+\gamma
$$

wcur. As was pointed out by Low, and discussed in more detail by Barut et al. $^{6}$, the simplest form for the electromagnetic coupling of the e to the $e^{*}$ in the Lagrangian (or the $\mu$ to the $\mu^{*}$ ) which obeys current conservation is?

$$
e\left(\frac{\lambda}{\mu+t}\right)\left[\sigma_{\mu \nu} F^{\mu \nu}+\text { h.c. }\right] \text {. }
$$

$\lambda$ is an unknown dimensionless constant which measures the relative strength of this special electromagnetic interaction compared to the conventional electromagnetic interaction. The mass of the heavy excited lepton, $M^{*}$, is inserted simply to make $\lambda$ dimensionless. Equation 4 means that, an $\mathrm{e}^{-}-\mathrm{e}^{*^{-}}$-photon vertex in a momentum space Feymman diagram has the form

$$
v^{\mu}=-e\left(\frac{\lambda}{M *}\right) \bar{u} \sigma^{\mu \nu} \quad q^{u}
$$

with $q$ the momentum of the photon (as shown in Fig. Ib), rather than the form

$$
\mathrm{v}^{\mu}=-i \text { e } \bar{u} \gamma^{\mu} \mathbf{u}
$$

of a conventional $\mathrm{e}^{-}-\mathrm{e}^{-}-$photon vertex

It is convenient and perhaps stimulating to the imagination to think of the $e^{*}$ and $\mu^{*}$ as the respective excited states of the $e$ and $\mu$. Hence we designate the $e^{*}$ and $\mu^{*}$ by the term heavy excited lepton. We may also consider the existence of a neutral heavy excited lepton, namely the heavy neutrino, $v^{*}$, with the same lepton number as is possessed by the $\nu_{e}$ or the $\nu_{\mu}$. However, we shall not require that the $e^{*}$ and $\nu^{*}$, or the $\mu^{*}$ and $v^{*}$, occur in pairs. In discussing the heavy excited charged leptons, we shall generally use the $e^{*}$ as the example.

c.

Lipmanor $^{8}$ and others ${ }^{9-13}$ have suggested that a lepton, say the $e^{\prime}$, might, have the same lepton number as the e of the opposite electric charge. The lepton number scheme would be

$$
\begin{gathered}
e^{-}, v_{e}, e^{++}, \bar{v}_{e^{\prime}} \text { have } n=+1, \\
e^{+}, v_{e}, e^{e^{-}}, v_{e^{\prime}} \text { have } n=-1 . \\
-6-
\end{gathered}
$$


A special case of this hypothesis is the assumption that the $\mu$ and the e form such a pair, ${ }^{11-15}$ namely,

$$
\begin{aligned}
& e^{-}, v_{e}, \mu^{+}, \bar{v}_{\mu} \text { have } n=+1, \\
& e^{+}, \nu_{e}, H^{-}, v_{\mu} \text { have } n=-1
\end{aligned}
$$

In the last few years there has been a strong revival of interest in some special pairs in connection with unified gauge theories of electromagnetic and weak interactions. In these theories the four particle point interaction of the classic Fermi formulation of weak interactions, Fig. $2 a$, is replaced by the interaction in Fifs. $2 b$ :

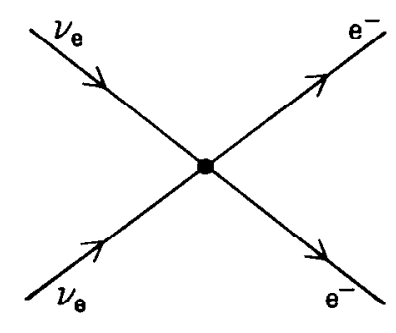

(a)

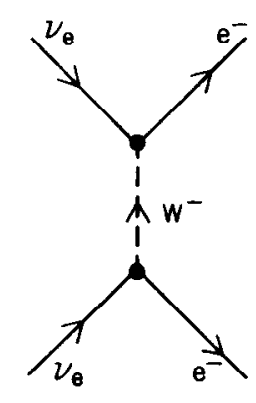

(b)
Fig. 2

The $\mathrm{W}^{ \pm}$is at present a hypothetical spin 1 boson with only weak and electromagnetic interactions. Its electron lepton number and muon lepton number are both zero. However, to prevent the non-physical energy dependence at very high energies of diagrams such as the one in Fig. 20 , and to make the theory renormalizable, additional particles must be hypothesized. Thus a neutral spin I boson analogous in properties to the $W^{+}$usually called the $Z^{\circ}$ may be assumed to exist. Either in addition to the $z^{\circ}$ or just by themselves, pairs of heavy leptons may also be assumed to exist. The following pairs of heavy leptons are usually assumed. ${ }^{17}$

$$
\begin{aligned}
& \mathrm{E}^{+}, \mathrm{E}^{\mathrm{O}} \text { with lepton numbers of } \mathrm{e}^{-}, \\
& \mathrm{M}^{+}, \mathrm{M}^{\mathrm{O}} \text { with lepton numbers of } \mu^{-},
\end{aligned}
$$

along with their antiparticles. To illustrate the need for such leptons and/or the $z^{\circ}$, as discussed in Refs. 17, these heavy leptors are used to cancel the unphysical high energy behavior of the diagram in Fig. 3a by means of the diagrams in Fig. 3b:

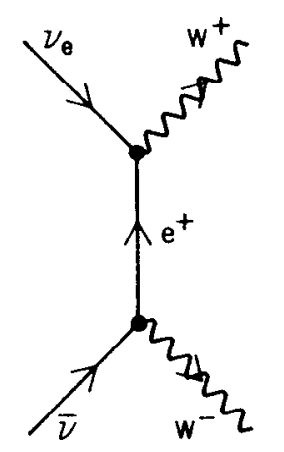

(a)

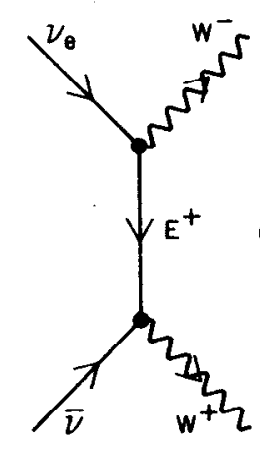

(b)

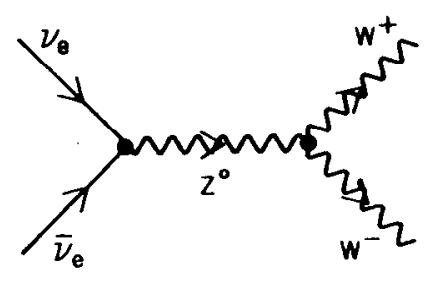

2590As 
For the experimenter, the most immediate significance of theories requiring $\mathrm{I}^{+}, \mathrm{E}^{\circ}$ and $\mathrm{M}^{+}, \mathrm{M}^{\circ}$ heavy leptons is that there is an additional incentive for searching for heavy leptons. Only if some aspects of these theories are verified--the existence of the $W$ being proven and its mass measured, for example--do these theories provide useful guides as to What might be the masses and properties of these leptons. 18,19

\section{Stable and Very Iong Lived Heavy Ieptons}

One may always assume that there is a special conservation rule or a special set of circumstances which give a heavy lepton a very long life or allows it to be stable. 20-22 Such special conditions are necessary to prohibit the decay processes which lead to the short lifetimes discussed in the next section. A simple way to obtain a stable heavy charged lepton is to assume that the lepton has a unique lepton number as in case of heavy sequential leptons, but to also assume that the associated neutrino has a nonzero mass which is greater than the mass of the charged lepton.

\section{E. Other Fossibilities}

Dbviously there are yet other possibilities for new types of heavy leptons, both charged and neutral. We have emphasized the four types described in II.A through II.D because they are convenient experimental classes upon which the discussion of the searches can be based.

\section{F. $\Lambda$ Word on Notation}

As an aid to the memory, we shall use the notation $\mu^{\prime}, \mu^{\prime \prime} \ldots$ to denote heavy sequential leptons; we shall use $e^{*}$ and $\mu^{*}$ to denote heavy excited leptons; and we shall use the script $l$ to denote any type of heavy lepton.

$$
-9-
$$

III. THE DECAY PROPERTIES OF THE HEAVY LFPTONS

\section{A. Charged Heavy Sequential Leptons}

The charged heavy sequential lepton will decay, through the weak interactions, in the leptonic modes

$$
\begin{aligned}
& \mu^{\prime} \rightarrow \nu_{\mu^{\prime}}+e^{-}+\bar{v}_{e}, \\
& \mu^{\prime} \rightarrow v_{\mu^{\prime}}+\mu^{-}+\bar{v}_{\mu} ;
\end{aligned}
$$

and, depending on the $\mu^{\prime}$ mass, in the hadronic modes

$$
\begin{aligned}
& \mu^{1^{-}} \rightarrow v_{\mu^{\prime}}+\pi^{-}, \\
& \mu^{1^{-}} \rightarrow v_{\mu^{\prime}}+K^{-},
\end{aligned}
$$

and

$$
\mu^{\prime}-\rightarrow v_{\mu^{\prime}}+2 \text { or more hadrons }
$$

In all of those reactions we ascume that the $\nu_{\mu^{\prime}}$ mass is sufficiently small to allow the decay to occur. The decay rates $(\Gamma)$ for the leptonic modes, Eqs. 8 and 9, have been discussed by many authors. 17,23-29 The calculation is straightforward if conventional, first order, weak interaction theory is used. The Feynman diagram for the decay is given in Fig. $4 a$

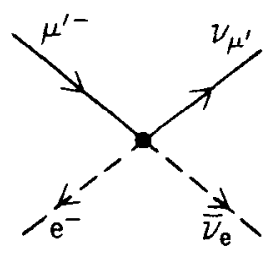

(a)

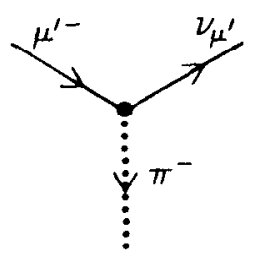

(b)
Fig. 4 258044 
where the solid and dashed lines distinguish the $n_{\mu^{\prime}}=+1$ lines from the $\mathrm{n}_{\mathrm{e}}= \pm \pm_{1}$ lines.

We find for

$$
\begin{aligned}
& M_{\mu^{\prime}} \gg M_{\mu}, \quad M_{\mu^{\prime}}=0 \\
& \Gamma\left(\mu^{\prime}-\rightarrow \nu_{\mu^{\prime}}+\mu^{-}+\bar{\nu}_{\mu}\right) \approx \Gamma\left(\mu^{\prime-} \rightarrow \nu_{\mu^{\prime}}+\mathrm{e}^{-}+\bar{\psi}_{\mathrm{e}}\right)=\frac{G^{2} M_{\mu^{\prime}}^{5}}{192 \pi^{3}}
\end{aligned}
$$

where

$$
G=1.02 \times 10^{-5} / \mathrm{M}_{\mathrm{p}}^{2}
$$

and $M_{p}$ is the mass of the proton. That is, the decay rate for the leptonic decay modes is given by the same formula as is used for the $\mu^{-} \rightarrow \mathrm{e}^{-}+\nu_{\mu}+\bar{v}_{\mathrm{e}}$ decay; the $\mu^{\prime}$ mass simply being substituted for the $\mu$ mass.

Two variations of Eq. 13 may occur:

(a) The $\mu^{\prime}$ may have a different weak interaction coupling constant frow that of the $\mu$ or e. Ur, more generally, there may be two different coupling constants corresponding to the vector and axial vector interactions of the $\mu^{\prime}$. Therefore, the effective $\mathrm{G}^{2}$ in Eq. 13 may not have the value given in Eq. 14. But we do not expect the effective $G^{2}$ to be different by more than a factor of 2 or 3 .

(b) If the mass of the $\mu^{\prime}$ neutrino $\left(M_{\mu^{\prime}}\right)$ is not zero, the decay rate will be smaller. ${ }^{17}$ Setting $z=M_{\mu^{\prime}} / M_{\mu^{\prime}}$, the right hand side of Eq. 13 will be multiplied by a function ${ }^{17}$ of $z$ which contains one or more powers of $\left(1-z^{2}\right)$.
The single hadron decay modes, Eq. 10 and 11 , can also be understood using conventional weak interaction theory. ${ }^{23}$ Thus the decay rate for $\mu^{\prime} \rightarrow \nu_{\mu^{\prime}}+\pi^{-}$is calculated using the diagram in Fig. 4b; the strength of the weak interaction coupling of the $\pi$ being obtained from $\pi^{-} \rightarrow \mu^{-}+\bar{v}_{\mu}$.

When the mass of the charged sequential lepton exceeds $1 \mathrm{GeV} / \mathrm{c}^{2}$ or so, the multi-hadron decay modes, Eq. 12, become important--perhaps most important. Yet it is just these modes which we know least how to calculate. We don't know how to calculate the cross hatched region in Fig. 5 a

from basic principles--not even in conventional weak interaction theory.

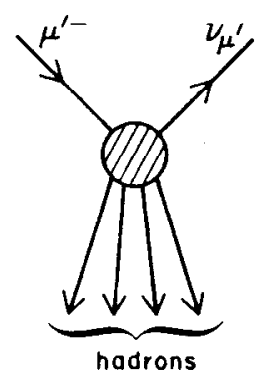

(a)

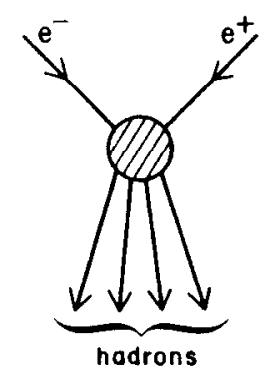

(b) 2580AS 
However, if we accept the conserved vector current hypothes is--a concept which connects some aspects of weak interactions with electromagnetic interactions--then there is a relation between

$$
\mu^{\prime}{ }^{-} \rightarrow Y_{\mu^{\prime}}+\text { hadrons }
$$

and

$$
\mathrm{e}^{-}+\mathrm{e}^{-} \rightarrow \text { hadrons }
$$

The reaction in Eq. 15b, the production of hadrons by positron-electron annihilation, is shown diagramically Fig. $5 \mathrm{~b}$.

The conserved vector

current hypothesis states that there is a single hadronic transition current with vector spatial properties whose charge changing component is the I (isospin) = l, strangeness conserving, part of the weak hadronic current and whose charge conserving part is the $I=1$ part of the electromagnetic hadronic current. Redrawing Fig. 5 to emphasize the hadronic transition current; Fig. 6 shows that we can connect the weak decay process of Fq. $15 \mathrm{a}$ to the electromagnetic reaction Eq. $15 \mathrm{~b}$.

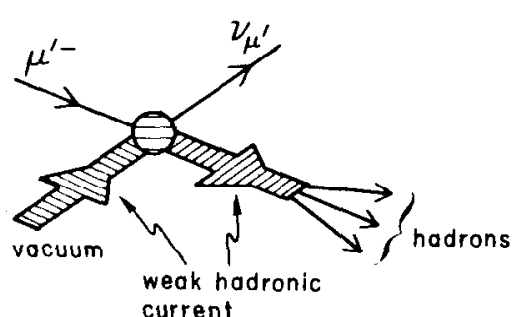

(a)

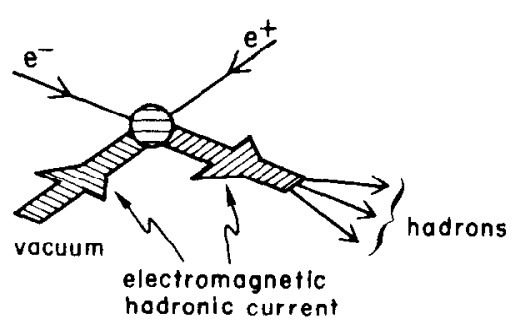

(b)

238040
The quantitative relation in Eq. 16 has been given by Tsai, ${ }^{23}$ using some additional assumptions about the contributions of $I=0$, axial vector, and strangeness changing currents.

$$
\begin{aligned}
& \Gamma\left(\mu^{\prime} \rightarrow v_{\mu^{\prime}}+\text { hadrons }\right) \approx \frac{3 G^{2}}{2^{8} \pi^{4} \alpha^{2} M^{3}} \int \frac{M^{2}}{s_{0}}\left[s\left(M^{2}-s\right)^{2}\left(M^{2}+2 s\right)\right. \\
& \left.\times \quad \sigma_{e^{+} e^{-} \rightarrow \text { had }}(s)\right]
\end{aligned}
$$

Here $\sigma \mathrm{e}^{+} \mathrm{e}^{-} \rightarrow$ had $^{(3)}$ is the total cross section for the reaction $\mathrm{e}^{+}+\mathrm{e}^{-} \rightarrow$ hadrons when $\sqrt{\mathrm{s}}$ is the total energy in the center of mass. $M$ is the mass of the $\mu^{\prime}$. $\sqrt{s_{0}}$, the smallest invariant mass of hadrons which contribute to the decay $\mu^{\prime} \rightarrow \nu_{\mu^{\prime}}+$ hadrons, is taken to be about $1 \mathrm{GeV} / c^{2} . \alpha$ is the fine structural constant ( $1 / 137)$.

In thinking about $\sigma_{\mathrm{e}^{+}} \mathrm{e}^{-} \rightarrow$ had $(\mathrm{s})$, it has become conventional to relate it to the cross section $\mathrm{O}^{+} \mathrm{e}^{-} \rightarrow \mathrm{\mu}^{+} \mathrm{\mu}^{-}$(s) for the reaction $e^{+}+e^{-} \rightarrow \mu^{+}+\mu^{-}$by defining

$$
R(s)=\frac{\sigma_{e^{+} e^{-} \rightarrow \text { had }}(s)}{\sigma_{e^{+} e^{-} \rightarrow \mu^{+} \mu^{-}}(s)}
$$

using

$$
\sigma_{e}^{+} e^{-} \rightarrow \mu^{+} \mu^{-(s)}=\frac{4 \pi \alpha^{2}}{3 s}, s \gg M_{\mu}^{2}
$$

and Eq. 13, Fq. 16 take the simple furm

$$
\begin{aligned}
\frac{\Gamma\left(\mu^{\prime} \rightarrow \nu_{\mu^{\prime}}+\text { hadrons }\right)}{\Gamma\left(\mu^{\prime}-\nu_{\mu^{\prime}}+e^{-}+\bar{v}_{\mathrm{e}}\right)} & \approx 3 \int_{1 / \mathrm{M}^{2}}^{1} \mathrm{dx}\left[\left(1-\mathrm{x}^{2}\right)(1+2 \mathrm{x}) \mathrm{R}(\mathrm{s})\right] \\
\mathrm{x} & =s / \mathrm{M}^{2}
\end{aligned}
$$

Fig. 6 
Finally defining

$$
\overline{\mathrm{R}}=\int_{1 / \mathrm{M}^{2}}^{l} \mathrm{dx}\left[(1-\mathrm{x})^{2}(1+2 \mathrm{x}) \mathrm{R}(\mathrm{s})\right] / \int_{1 / \mathrm{M}^{2}}^{1} \mathrm{dx}\left[(1-\mathrm{x})^{2}(1+2 x)\right]
$$

we obtain

$$
\frac{\Gamma\left(\mu^{\prime-} \rightarrow v_{\mu},+ \text { hadrons }\right)}{\Gamma\left(\mu^{\prime}{ }^{-} \rightarrow v_{\mu},+e+\bar{v}_{e}\right)} \approx \frac{3}{2} \bar{R}
$$

(s) has now been measured ${ }^{30,31}$ for $\sqrt{s} \leq 5 \mathrm{GeV}$. A rough fit to Eq. 17 yields (for s in units of $\mathrm{GeV}^{2}$ )

$$
R(s) \approx \frac{5}{4} \sqrt{s}-\frac{3}{4}, 1 \mathrm{GeV} \leq \sqrt{\mathrm{s}} \leq 5 \mathrm{GeV}
$$

Putting all this together we obtain the fractional decay rates in Fig. 7, and the lifetime in Fig. 8. Here we have assumed

$$
R(s)=5.5, \sqrt{s}>5 \mathrm{GeV}
$$

In Fig. 7 we note the dominance of the multi-hadron decay mode.

To remind us how speculative this is, we also show the fractional decay rates, Fig. 9, if we use a quark-parton model 23 in which

$$
R(s)=\text { constant }=2 / 3
$$

This model, gives the wrong value of $R$ as measured in $\mathrm{e}^{+}+\mathrm{e}^{-} \rightarrow$ hadrons.

$R$ will turn out to be a useful concept when discussing methods of searching for heavy leptons. However we don't know if the preceeding theory connecting the multi-hadron decay mode of a heavy lepton with the reaction $\mathrm{e}^{+}+\mathrm{e}^{-} \rightarrow$ hadrons is correct. Therefore in analogy to Eq. 20 we define for any hypothetical charged sequential lepton

$$
\frac{\Gamma\left(\mu^{\prime-} \rightarrow \nu_{\mu^{\prime}}+\text { hadrons }\right)}{\Gamma\left(\mu^{\prime-} \rightarrow \nu_{\mu^{\prime}}+e+\bar{\nu}_{e}\right)}=\frac{3}{2} R_{\text {decay }}
$$

If the preceeding theory is correct $R_{\text {decay }}=\vec{R}$, otherwise we are in lieu of other knowledge free to choose any value for $R_{\text {decay }}$.

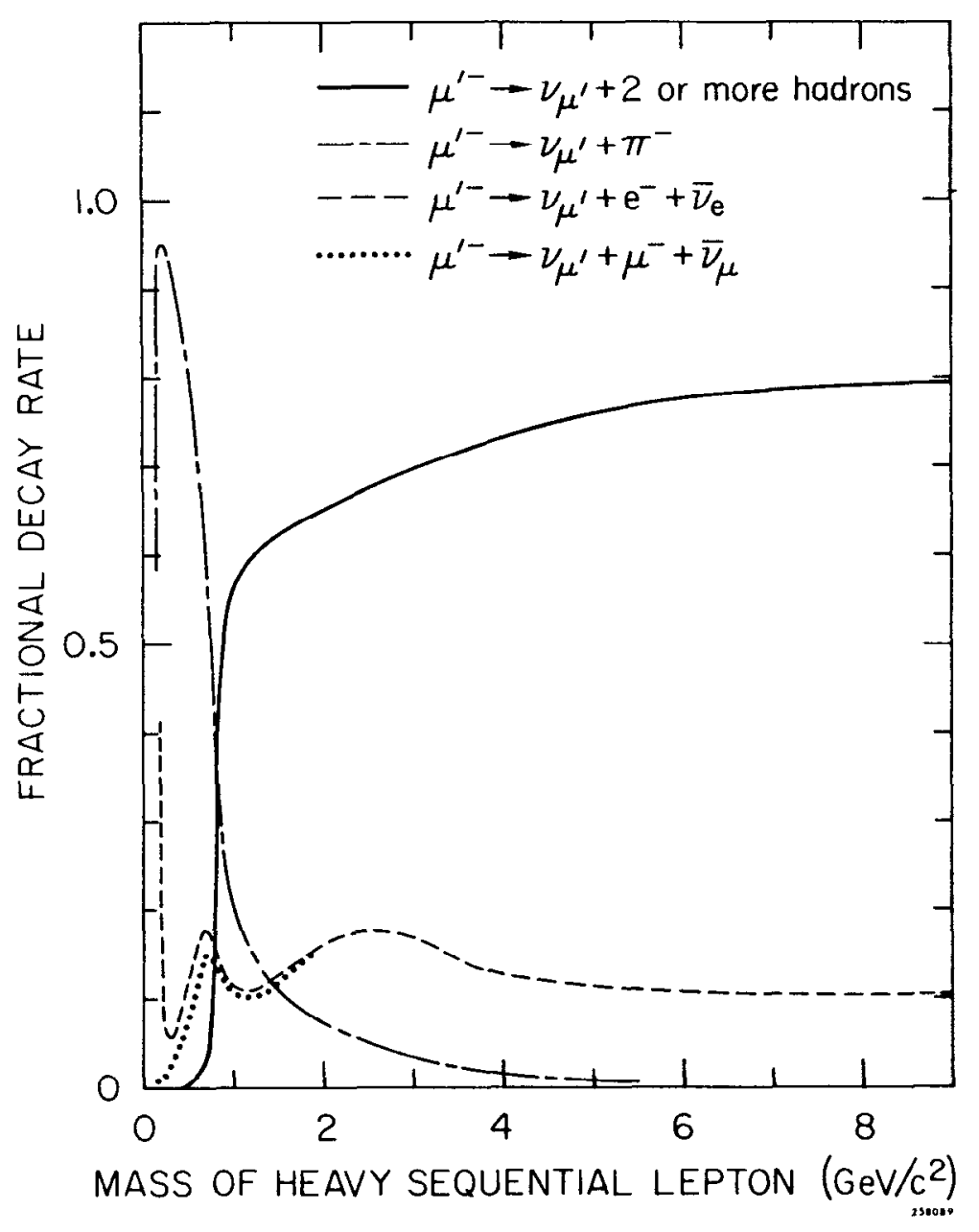

Fig. 7. Fractional decay rates of heavy sequential leptons based on calculations discussed in the text with $\mathrm{R}$ given by Eq. 21. The $v_{1}$, mass is assumed to be zero. The low mass behavior of the 2 or more hadrons decay mode has been smoothed over the individual decay channel thresholds.

$$
-15 \text { - }
$$




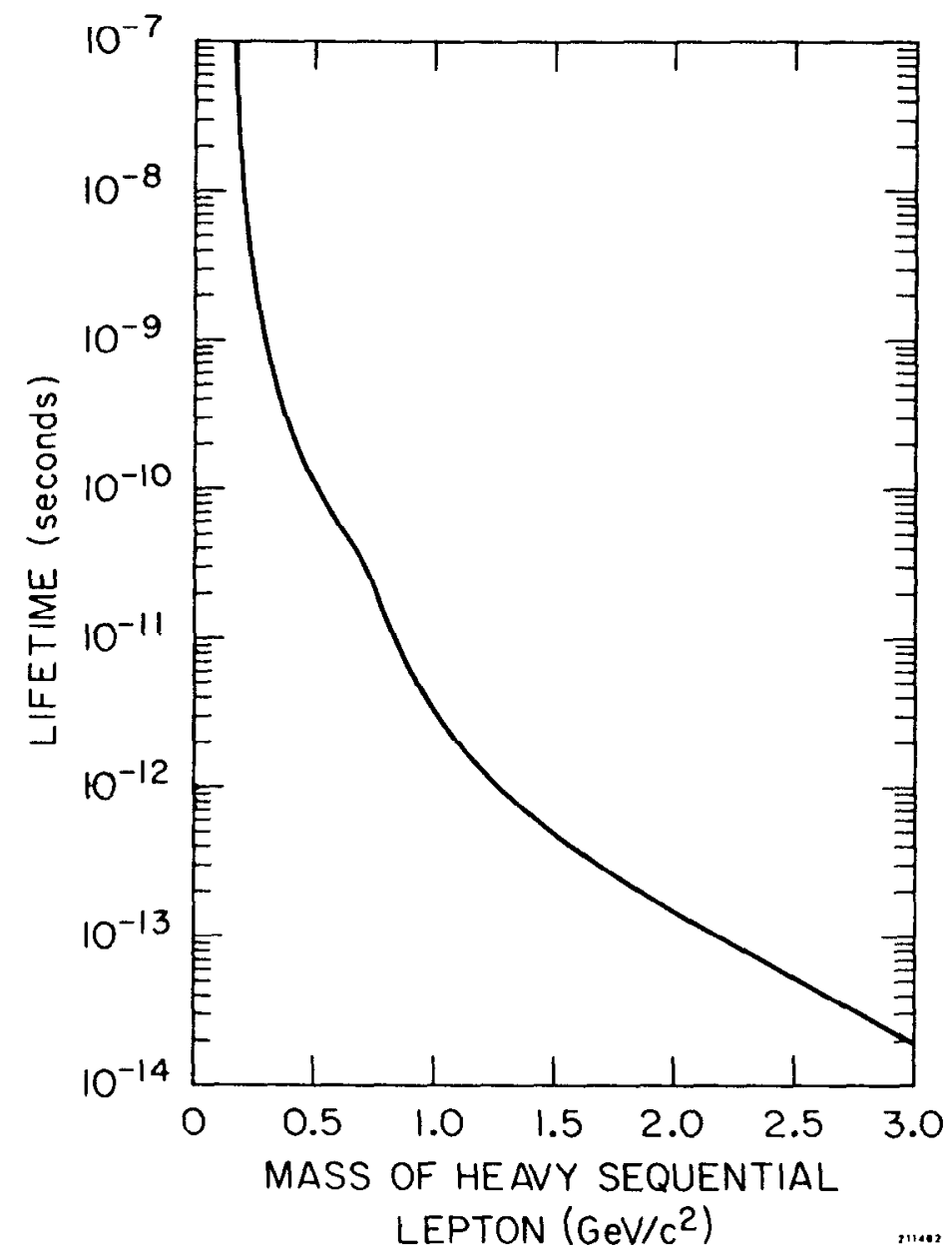

fig. 8. Lifetime versus mass of heavy sequential leptons based on calculations discussed in the text using Eq. 21 and zero mass for the $v_{\mu}$,

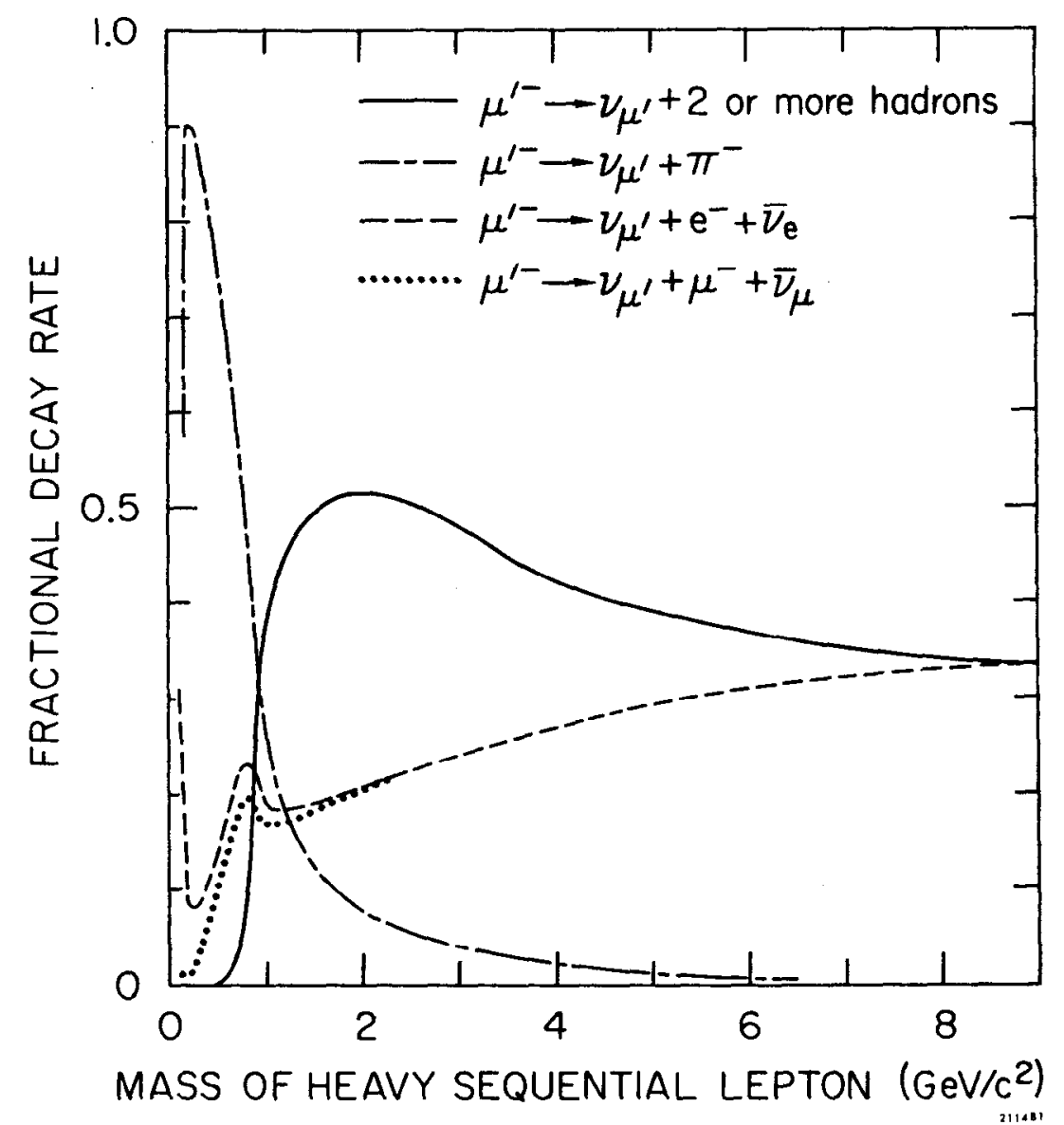

Fig. 9. Fractional decay rates of heavy sequential leptons based on calculations discussed in the text using $R=2 / 3$ as in Eq. 22 . The mass of the $v^{\prime}$ is assumed to be zero. The low mass behavior of the 2 or more hadrons decay modes has been smoothed over the individual decay channel thresholds. 
In this discussion we have ignored the possibility of the existence of a charged intermediate boson $W$ which mediates the weak interactions. If the mass of this hoson $M_{W}$ is less than $M_{\mu^{\prime}}$, the decay rate of the process

$$
\mu^{\prime^{-}} \rightarrow \nu_{\mu^{\prime}}+\mathrm{W}^{-}
$$

will be much greater than the processes we have considered. This is easily seen as follows. The decay rate for this process is 23

$$
\Gamma\left(\mu^{\prime} \rightarrow \nu_{\mu^{\prime}}+W^{-}\right)=G_{\mu^{\prime}}^{3}\left[\frac{\left(1-r^{2}\right)^{2}(1+2 r)}{8 \pi \sqrt{2}}\right]
$$

where

$$
r=\left(M_{W}^{2} / M_{\mu^{\prime}}^{2}\right)<1 .
$$

Comparison of Eq. 25 with Eq. 14 yields the approximate ratio

$$
\frac{\Gamma\left(u^{\prime} \rightarrow v_{\mu^{\prime}}+W^{-}\right)}{\Gamma\left(\mu^{\prime} \rightarrow \nu_{\mu^{\prime}}+e^{-}+\bar{v}_{e}\right)} \sim \frac{1}{G_{\mu^{\prime}}} \sim 10^{5}\left(\frac{M_{p}}{M_{\mu^{\prime}}}\right)^{2}
$$

Therefore, if $M_{W}<M_{\mu}$, the observed decay modes of the $\mu^{\prime}$ will be just the decay modes of the $W$. Since the $W$ is still a hypothetical particle, we can say little about the details of its hadronic modes. But we can apply to the decay $\mathrm{W}^{-} \rightarrow$ hadrons the same general considerations which led to Eq. 18 . Therefore, we expect that the $\mu^{\prime}$ and the $W$, if their masses are in the several $\mathrm{GeV} / \mathrm{c}^{2}$ range or larger, will have roughly similar 2 or more hadron decay modes and roughly similar ratios of that mode to the leptonic modes. Thus the search experiment that first finds the $\mu^{\prime}$ will probably not show if the intermediate boson forms a step in the decay chain through the process of Eq. 24.
The lifetime of the $\mu^{\prime}$ will of course depend upon whether or not there is an intermediate bosun $W$ with $M_{W}<M_{\mu^{\prime}}$. In F1g. 8 we show the lifetime of the $\mu^{\prime}$ assuming that there is no $W$ with $M_{W}<M_{\mu^{\prime}}$ and using Eqs. 13 through 21 . If such a $W$ exists, the $\mu^{\prime}$ has the very short lifetime

$$
\tau \approx 2.5 \times 10^{-23}\left(\frac{M_{p}}{\frac{M_{\mu^{\prime}}}{3}}\right)^{3}\left[\frac{1}{\left(1-r^{2}\right)^{2}(1+2 r)}\right] \text { sec . }
$$

\section{B. Neutral Heavy Sequential Leptons}

If the mass of the neutral heavy sequential lepton $\left(M_{\mu^{\prime}}\right)$, that is of the neutrino, is less than that of the charged lepton $\left(M_{\mu^{\prime}} \pm\right)$, as was assumed in the previous section, then like the conventional neutrinos the $\nu_{\mu^{\prime}}$ is stable! A most peculiar object if $M_{\mu^{\prime}}$ is large.

$$
M_{\mu^{\prime}}>M_{\mu^{\prime}} \pm
$$


then the $v_{\mu}$, has decay modes completely analogous to that in the previous section:

$$
\begin{aligned}
& \nu_{\mu^{\prime}} \rightarrow \mu^{\prime-}+\mathrm{e}^{+}+\nu_{\mathrm{e}} \\
& \nu_{\mu^{\prime} \rightarrow \mu^{\prime}}+\pi^{+} \\
& \nu_{\mu^{\prime} \rightarrow \mu^{\prime}}+2 \text { or more hadrons }
\end{aligned}
$$

and so forth. The decay ratio will be the same as those for the analogous $\mathrm{H}^{\prime-}$ decay modes given in the previous section. But now, we remind the reader, the $\mu^{\prime}$ will be stable! An even more peculiar object :

\section{Special Pairs of Leptons}

The decay mode calculations for special pairs of leptons ${ }^{17}$ are, in many ways, similar to those for heavy sequential leptons. To illustrate the discussion, we use the $\mathrm{E}^{+}, \mathrm{E}^{\circ}$ pair which has the lepton numbers of the $\mathrm{e}^{-}$.

If

$$
\mathrm{M}_{\mathrm{E}}+>\mathrm{M}_{\mathrm{E}} \circ
$$

analogous to Eqs. 8 and 12 , we have

$$
\begin{aligned}
& E^{+} \rightarrow E^{0}+e^{+}+v_{e} \\
& E^{+} \rightarrow E^{0}+2 \text { or more hadrons }
\end{aligned}
$$

Unlike the sequential heavy Iepton even if $\mathrm{M}_{\mathrm{E}}+<\mathrm{M}_{\mathrm{E}}{ }^{\circ}$, the $\mathrm{E}^{+}$is unstable because there are the decay modes

$$
\begin{aligned}
& \mathrm{E}^{+} \rightarrow \nu_{e}+\mathrm{e}^{+}+v_{\mathrm{e}} \\
& \mathrm{E}^{+} \rightarrow \nu_{\mathrm{e}}+2 \text { or more hadrons }
\end{aligned}
$$

The $\mathrm{E}^{\mathrm{O}}$ is also always unstable, because of the decay modes

$$
\mathrm{E}^{\mathrm{O}} \rightarrow \mathrm{e}^{-}+\text {(leptons or hadrons) }
$$

as well as the modes (when $M_{E} \circ>M_{E}+$ )

$$
\mathrm{E}^{\circ} \rightarrow \mathrm{E}^{+}+\text {(leptons or hadrons) }
$$

and since neutral weak interaction currents exist we can also expect

$$
\mathrm{E}^{\mathrm{O}} \rightarrow v_{\mathrm{e}}+\text { (leptons or hadrons) }
$$

The decay rates for the $\mathrm{E}^{+}, \mathrm{E}^{\circ}$ have been discussed by Bjorken and Llewellyn Smith ${ }^{17}$. The calculational methods and the predictions are similar to those given for the sequential heavy leptons. For crude estimates, Figs. 7 through 9 can be used.

D. Charged Heavy Excited Leptons

The very dominant decay mode for the charged heavy excited leptons

is

$$
e^{*} \rightarrow e+\gamma ;
$$

all other decay modes have ratios which are smaller by at least the factor $\alpha^{2}$. The Iifetime for $M_{e^{*}} \gg M_{e}$ is 32

$$
\tau=\frac{1}{\alpha M_{e^{*}} \lambda^{2}}=\frac{.9 \times 10^{-22}}{M_{e} \lambda^{2}} \mathrm{sec},
$$

where $M_{e} *$ is in $\mathrm{GeV} / \mathrm{c}^{2}$.

$$
\text { IV. SEARCHES FOR IOW MASS OR STABLE HEAVY LEPTONS }
$$

When the mass of the heavy lepton is sufficiently small, there are two convenient search methods. First, if the charged or neutral heavy lepton has a mass $M_{l}$ less than the pion mass $M_{\pi}$ or kaon mass $M_{K}$, the heavy lepton $l$ can appear in the decay of the pion or kaon respectively. Second, for heavy sequential leptons, a small mass leads to a Iifetime sufficiently long to permit detection if the lepton is in a particle beam of conventional 
length--namely tens of meters.

A. Searches in the Decay Modes of the Fion and Kaon

1. The Method

Most past searches of this type have involved direct observation in a bubble chamber, but counters have been used. ${ }^{33}$ Some recent tests of the Ramm effect ${ }^{34}$ discussed below use wire spark chambers. There is no uniform method and the reader should consult the references which are cited in this section.

2. Past Searches

With one exception, which will be discussed below, no evidence for heavy leptons, either charged or neutral has been found in the study of the decay modes of the pion or kaon. Rothe and Wolsky ${ }^{29}$ summarized the situation in 1968. An earlier summary is provided by Beier, ${ }^{33}$ who carried out a search for heavy sequential leptons with masses just below the kaon mass.

The exception to these null results is the work of $\mathrm{amm}^{34}$ who reports evidence for a neutral heavy meson in the decay $\mathrm{K}_{\mathrm{L}}^{\mathrm{O}} \rightarrow \mu^{ \pm}+\pi^{+}+\nu_{\mu}$. The heavy lepton appears as a narrow resonance in the $\mu^{\circ *} \rightarrow \mu^{ \pm} \pi+$ mass spectrum with mass $.422<M_{\mu \pi}<.437 \mathrm{GeV} / \mathrm{c}^{2}$. Ramm ${ }^{34}$ also reports evidence in neutrino interactions and in muon bremsstrahlung 35,36 for a charged counterpart with the decay mode $\mu^{*} \rightarrow \mu^{-}+\gamma$. Unfortunately, this work has not been verified by other studies of the $K_{L}^{0}$ decay spectrum. While most of these studies with null results have not been published, Clark et al. ${ }^{37}$ have published a very high statistics study, also reporting a null result. The weight of the evidence appears at present to be against the existence of the Ramm neutral heavy lepton.
3. Future Searches

We know of no plans for further searches for heavy leptons in pion or kaon decay modes.

B. Searches in Particle Beams for Short-Iived Charged Heavy Leptons

1. The Method

For a charged heavy lepton to be directly detected in a particle bear:, the lepton's decay length in the laboratory frame must be of the order of magnitude of, or greater than, say 10 meters. This requires a laboratory decay time of at least $3 \times 10^{-8}$ seconds. If the particle has a laboratory frame energy $E_{\ell}$, the time dilation factor $\left(E_{\ell} / M_{l}\right)$ may permit detection of particles with particle rest frame lifetimes $\left(\tau_{\ell}\right)$ as short as $\left(M_{\ell} / E_{l}\right)\left(3 \times 10^{-8}\right)$ seconds.

Searches at electron accelerators are most useful because, as is discussed in Sec. V.C, the heavy leptons are pair produced by photons witr. a known cross section, if they have unit charge. Searches at proton accelerators are less definitive, because the production cross section of the heavy leptons is not known; this production uncertainty is discussed in Sec. V.D.

2. Past Searches

Bxamples of searches at electron accelerators all with null results are the experiment of Coward et $21 .{ }^{38}$ who searched in the mass range of .5 to $90 \mathrm{MeV} / \mathrm{c}^{2}$, and the experiment of Barna et al. ${ }^{21}$ who studied the mass range 39 of .2 to $1.0 \mathrm{GeV} / \mathrm{c}^{2}$. Because of their uncertain sensitivity, searches at proton accelerators are usually not reported, although we may 
be sure that any unknown charged particle which was found in a beam would have been reported. We can set a crude upper limit to the mass range of these searches by noting that at the older proton accelerators $\left(\mathrm{M}_{\ell} / \mathrm{E}_{\ell}\right) \gtrsim .01$. Therefore, ${ }^{\tau} \gtrsim 3 \times 10^{-10}$ seconds and, from Fig. $8, M_{\ell} \lesssim 35 \mathrm{GeV} / \mathrm{c}^{2}$.

In closing this section we note an experiment of Ansorge et al. 40 in which a search was made for charged and neutral particles with masse less than $.1 \mathrm{GeV} / \mathrm{c}^{2}$. The search method involved the study of electron pair production and electron-like bremsstrahlung in a hydrogen bubble chamber. No new particles were found. 40

3. Future Searchea

We know of no special plans for future direct searches for low mass, short-lived, heavy leptons. The problem is that the new higher energy accelerators do not enlarge substantially the mass range over which charged heavy sequential leptons can be directly found in particle beams. For example, increasing $E_{l}$ by a factor of 10 decreases the lower limit on $T_{l}$ by a factor of 10 . But since $\tau$, decreases at least as fast as $\mathrm{M}_{l}^{-5}$, this only extends the upper limit on $\mathrm{M}_{l}$ to $10^{1 / 5}(.35)=.56 \mathrm{GeV} / \mathrm{c}^{2}$.

C. Searches for Stable Charged Heavy Ieptons in Particle Beams

1. The Method

The only question with respect to this type of search is whether its sensitivity is sufficient. Assuming unit charge for the leptons, this sensitivity can be determined quite well for particle beams produced by electrons or photons, Sec. V.C.; but for beams produced by protons, the very uncertain concepts in Sec. V. $P$. result in an indeterminate sensitivity
2. Past Searches

No stable heavy leptons have been found. At electron accelerators the search has been conducted ${ }^{21,22}$ with sufficient sensitivity up to a heavy lepton mass of $1.0 \mathrm{GeV}$. References 41 are exampies of older searches at proton accelerators. Recent searches ${ }^{42-44}$ include that of B. Alper et al. ${ }^{42}$ at the CERN $30 \mathrm{GeV}$ proton-proton colliding beams facility; and that of J. W. Cronin et al. ${ }^{43}$ using a $300 \mathrm{GeV}$ primary proton beam on a copper target at NAL. In the latter search, no charged heavy lepton was found in an equivalent yield of $10^{9}$ pions.

3. Future Searches

We assume that everyone who is studying the spectra of particles produced at any of the new accelerators will continue to search for stable heavy leptons, but with steadily decreasing enthusiasm. We also note that it is quite easy to look for stable charged heavy leptons at electronpositron colliding beam facilities.

V. SEARCHES FOR UNSTABLE HEAVY LEPTONS WITH MASSES GREATER THAN ABOUT $.5 \mathrm{GeV} / \mathrm{c}^{2}$

\section{A. Detection Method}

In the last section we showed that the search for heavy leptons with masses less than .4 or $.5 \mathrm{GeV} / \mathrm{c}^{2}$ was just about complete. And we showed that no heavy leptons with masses less than .4 or $.5 \mathrm{GeV} / \mathrm{c}^{2}$ have been proven to exist. Therefore the future of heavy lepton searches belongs to the mass range above $.5 \mathrm{GeV} / \mathrm{c}^{2}$. As discussed in Sec. III, heavy sequential leptons, special pairs, and of course heavy excited leptons, above this mass will have lifetimes less than $10^{-9}$ or $10^{-10}$ seconds. These heavy leptons must, 
thcrcfore, be detected through their decay products. And this detection must be carried out in the midst of a relatively large background of particles whtch have nothing to do with the heavy lepton production reaction. The same initial states, such as $\mathrm{e}^{+}+\mathrm{e}^{-}$or $\mathrm{p}+\mathrm{p}$, which can lead to heavy lepton production also lead to much more copious hadron productior:

In searches for heavy sequential leptons or for special lepton pairs, most detection metrods make use of special properties of one or more of the decay modes.

For example, the production of heavy sequential leptons or of special pairs will be relatively easy to observe if the leptonic decay modes can be ised. The clearest signature is a $\mu$ e pair with no ol,her charged particies present; the $\mu$ comes from the decay of one heavy lepton, the e comes from the decay of the other heavy lepton. Although there are some background problems, $\mu \mu, \pi \mu$, or $\pi$ pairs with no other charged particles present also provide good signatures; the $\pi$ coming from the $l \rightarrow v+\pi$ decay mode. We will mention later other detection methods which depend upon the $e$ or $\mu$ momentum spectrum. Of course, the sensitivity depends upon the fractional decay rates to these single chrged particle states. As $R_{\text {decuy }}$, Eq. 23, increases, the sensitivity of this method decreases.

Indeed if $R_{\text {decay }}$ is as large as 10 or so, the detection of the leptonic or single $\pi$ decay modes becomcs very difficult. One might hope then to make use of the $\left(v_{\mathrm{e}}+2\right.$ or more hadrons) decay mode. But here nature is unusually cruel--there is no simple way to separate the multi-hadron decay modes from the hadronic background. This is the problem that plagues present day heavy lepton searches; we will come upon it. repeatedly in the remainder of this section.

Turning now to the search for excited leptons, the $e^{*}$ or $\mu^{*}$, the experimenter has the advantage that he seeks only the $(e+\gamma)$ or $(\mu+\gamma)$ decay modes. He has the disadvantage that:

(a) There are no other decay modes to confirm the existence of the $e^{*}$ or $\mu^{*}$.

(b) There are backgrounds which can simulate the desired signal. For example the process

$$
e^{+}+e^{-} \rightarrow e^{+}+e^{-*}, e^{-*} \rightarrow e^{-}+\gamma
$$

can be confused with radiative $\mathrm{e}^{+} \mathrm{e}^{-}$scattering

$$
e^{+}+e^{-} \rightarrow e^{+}+e^{-}+\gamma
$$

B. Electron-Positron Colliding Beams Production of Heavy Leptons

1. The Method

The production of charged heavy leptons pairs $\ell^{-}, \ell^{+}$in electronpositron colliding beams takes place through the reaction

$$
\mathrm{e}^{-}+\mathrm{e}^{+} \rightarrow \mathrm{l}^{-}+\mathrm{e}^{+}
$$

The dominant Feynman diagram for this process is given in Fig. 10i

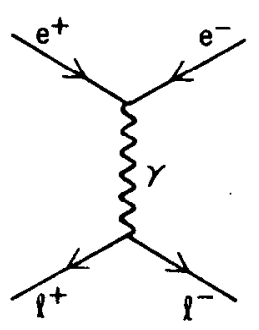

(a)

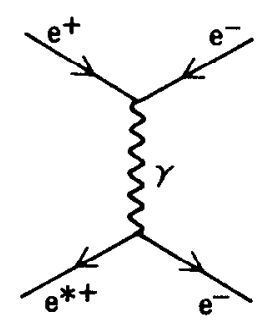

(b)
Fig. 10 258048 
Assuming the heavy leptons are Dirac point particles with unit charge, the total production cross section is

$$
\sigma\left(e^{-} e^{+} \rightarrow 1^{-} \ell^{+}\right)=\frac{\pi \alpha^{2}}{2 E^{2}} \beta\left[1-\beta^{2} / 3\right],
$$

where $E$ is the energy of either the electron or positron beam, and $\beta$ is the velocity of the $\ell$. We shall also use $s=4 E^{2}$.

Given that the electron-positron colliding beams have sufficient energy for lepton production, $E>M_{\ell}$, this method provides in our view the best general method of searching for charged heovy leptons. It has two

advantageous properties:

(a) The production cross section, Eq. 44 , is relatively large. Once $E$ is somewhat larger than $M_{1}, B$ approaches 1 , and

$$
\sigma\left(\mathrm{e}^{-\mathrm{e}^{+}} \rightarrow \ell^{-}{\ell^{+}}^{+}\right) \approx \sigma\left(\mathrm{e}^{-} \mathrm{e}^{+} \rightarrow \mu^{-} \mu^{+}\right) \approx \frac{2 \times 10^{-32}}{\mathrm{~F}^{2}} \mathrm{~cm}^{2},
$$

where $\mathrm{E}$ is in $\mathrm{GeV}$. Thus ${\ell^{-}}^{+}$pairs are produced almost as copiously as $\mu^{-}{ }^{+}{ }^{+}$pairs. Now we recall from Eqs. 17 and 21 that

$$
\sigma\left(\mathrm{e}^{-} \mathrm{e}^{+}-\mathrm{h} \text {.druns }\right)=-B(\mathrm{~s}) \sigma\left(\mathrm{e}^{-} \mathrm{e}^{+} \rightarrow \mu^{-} \mu^{+}\right)
$$

where

$$
1 \lesssim R \lesssim 5
$$

Therefore, the cross section for heavy lepton production is within a factor of 10 of the dominant hadron production cross section (Fig. 11). This is a much better situation than occurs in the production of heavy leptons by hadrons or photons iSces. V.C and V.D.).

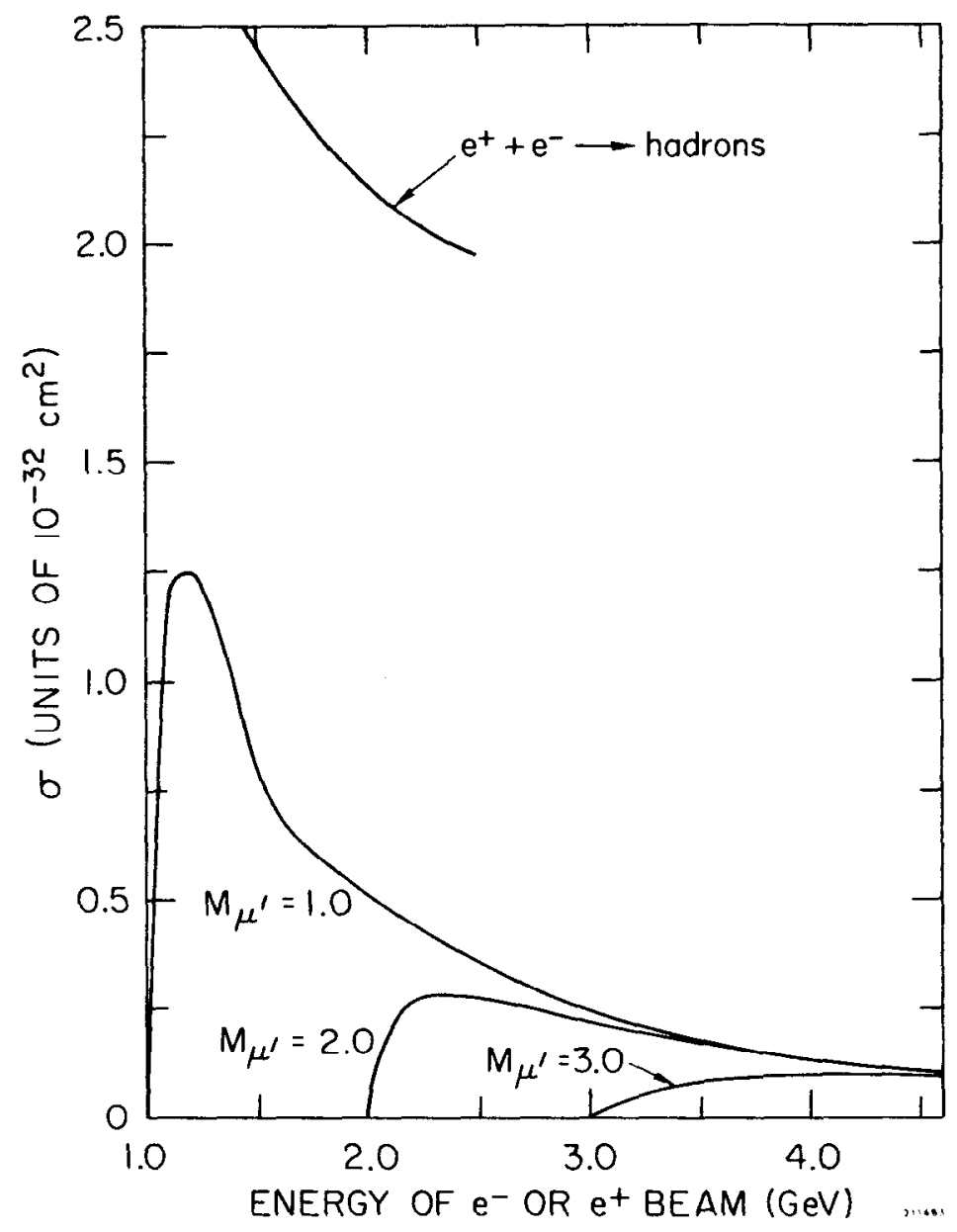

Fig. 11. Total cross section for the production of heavy leptons of mass $M$, at an electron-positron colliding beam facility, through the reaction $\mathrm{e}^{+}+\mathrm{e}^{-} \rightarrow \mu^{+{ }^{+}+\mu^{-}}$. For comparis on the uppermost solid. . Pror the production of in $\mathrm{e}^{+}+\mathrm{e}^{-} \rightarrow$ hadrons; based on Refs. 30 and 31 . 
(b) The lepton production cross section is relatively independent of the spin or electromagnetic moment properties of the $l$. Higher spins, anomalous magnetic moments or higher electromagnetic moments usually increase $e^{-} e^{+} \rightarrow \ell^{-} \ell^{+}$. The cross section will only be substantially smaller than that given in Eq. 46 , if the electric charge of the $l$ is much less than that of the electron, or if the $l$ has a form factor which is much less than unity for $q^{2}=4 \mathrm{E}^{2}$.

We should also remind you that for the excited electron, $e^{*}$, the limit on the heavy lepton mass is not $M_{e^{*}}<\mathrm{E}$, but is

$$
\text { for } \begin{gathered}
M_{\mathrm{e}^{*}}<2 \mathrm{~V} \\
\mathrm{e}^{+} e^{-} \rightarrow \mathrm{e}^{ \pm *}+\mathrm{e}^{\bar{f}} \\
\mathrm{e}^{ \pm^{*}} \rightarrow \mathrm{e}^{ \pm}+\gamma
\end{gathered}
$$

This can occur because the vertex in Eq. 4 allows the $e^{*}$ production diagram in Fig. 10b. In this case the production cross section of Eq. 47 will be reduced by roughly $\lambda^{2}$, and $\lambda$ is of course unknown. A similar result holds for the $\mu^{*}$.

\section{Past Searches}

A series of searches for charged sequential leptons has been carried out by V. Alles-Borelli et al. ${ }^{45}$ at ADONE; the $e^{+} e^{-}$colliding beams facility at Frascat1. These experimenters 20oked for $e^{ \pm} \mu^{\mp}$ pairs coming from the sequence

$$
\begin{aligned}
& e^{-}+e^{+} \rightarrow \mu^{\prime^{-}}+\mu^{\prime^{+}} \\
& \mu^{\prime} \rightarrow v_{\mu^{\prime}}+\mu^{-}+\bar{v}_{\mu} \\
& \mu^{\prime^{+}} \rightarrow \bar{v}_{\mu^{\prime}}+e^{+}+v_{e},
\end{aligned}
$$

or the alternative set of leptonic decay modes. No heavy leptons were found in the mass range of 0.2 to $1.0 \mathrm{GeV} / \mathrm{c}^{2}$. If the heavy leptons are assumed to couple only to leptons, the range extends to $1.4 \mathrm{GeV} / \mathrm{c}^{2}$.

J. Feller et al. ${ }^{46}$ conducted a search at the CEA $\mathrm{e}^{+} \mathrm{e}^{-}$colliding beans facility for charged sequential leptons with up to $2 \mathrm{GeV} / \mathrm{c}^{2}$ mass. They were not able to rule out the existence of one heavy lepton in this mass range. But assuming $R_{\text {dec } j y}=2$, they could show that the entire $e^{+} e^{-} \rightarrow$ hadrons cross section could not be due to the production of a series of heavy leptons.

Two searches 47,48 for excited heavy leptons hive been carried out using $e^{+} e^{-}$colliding beams. In the first search, ${ }^{47}$ conducted at Frascati, the reaction of $\mathrm{Eq}$. $49 \mathrm{~b}$

$$
e^{+}+e^{-} \rightarrow e^{+}+e^{-}+\gamma
$$

was analyzed and the limit of

$$
2 \Lambda_{e^{*}}=\frac{M^{*}}{\lambda} \geq 7.8 \mathrm{GeV}
$$

was obtained with a $95 \%$ confidence level.

The second search, 48 at CEA, focussed on deviations from the QEDO prediction for the reaction

$$
e^{+}+e^{-} \rightarrow \gamma+\gamma
$$

Two Feynman diagrams contribute, in the lowest order, to this process: 

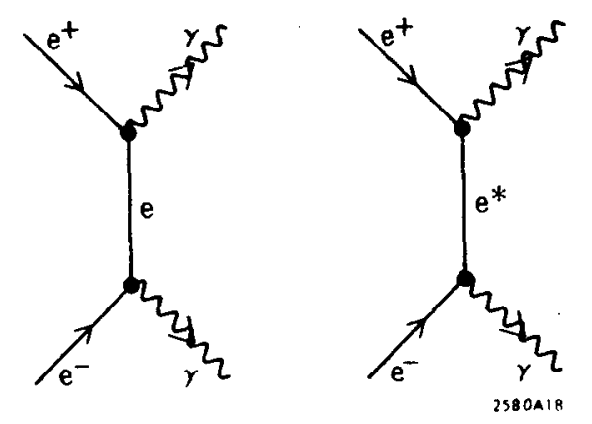

Fig. 12

An upper limit for $\lambda^{2}$ of $8 \times 10^{-5}$ to $9 \times 10^{-4}$ was set for a heavy electron with mass $M^{*}$ in the range of .6 to $2.4 \mathrm{GeV}$, or equivalently,

$$
2 \Lambda_{\mathrm{e}^{*}}=\frac{\mathrm{M}^{*}}{\lambda}>30 \text { to } 70 \mathrm{GeV}
$$

3. Present and Future Searches

The authors and their colleagues are now conducting a search ${ }^{49}$ for charged sequential and special pair leptons at SPEAR, the SLAC $e^{+} e^{-}$ colliding beams facility. SPEAR has operated with a maximum energy of $2.6 \mathrm{CeV}$ in each beam, and within a year the maximum energy will be increased to $4.8 \mathrm{GeV}$ in each beam.

To give a feeling for the sensitivity of such a search, we note that a good luminosity, ${ }^{50} \mathrm{I}$, is about 0.5 to $1.0 \times 10^{31}$ events per $\mathrm{cm}^{2}$ per sec. Using Eq. 47 we see that even for leptons with masses in the $4-5 \mathrm{GeV} / \mathrm{c}^{2}$ we expect ahout 10 heavy lopton pairs per hour. Of course, the problem is how to detect them. We are not as sanguine as we once were about using the multihadron decay mode, Eq. 12. It is true that this mode contributes to the total hadronic cross section. But the large values of $\mathrm{R}, \mathrm{Eq} . \quad 21$ and Fig. 11, make it difficult to detect the additional contribution to hadron production when the beam energy is above the lepton production threshold.

Therefore, the search makes use of the leptonic and single $\pi$ decay modes. When both members of the lepton pair decay into these modes, the final state shows only two charged particles and no $\pi^{\circ}, s$. Fven if there is misidentification of the $e, \mu$, or $\pi$, events with this signature are rare in the high multiplicity hadronic background.

Another way to use the leptonic or single $\pi$ decay modes is being pursued by J. Kirkby. ${ }^{51}$ In this method one uses the momentum imbalance in the event due to the undetected momentum carried off by the neutrinos.

Similar searches will no doubt be carried out at the DESY $\mathrm{e}^{+} \mathrm{e}^{-}$storage ring, DORIS, which will have a maximum energy of $5 \mathrm{GeV}$ in each beam.

The major limitations on the electron-proton colliding beam search method is that, except for the special case of Eq. $45, \mathrm{M}_{\ell}<\mathrm{E}$ and $\mathrm{E}$ is less than $5 \mathrm{GeV}$ for existing facillties. In contrast, as discussed in Sec. V.C., a $200 \mathrm{GeV}$ photon beam at a $300 \mathrm{GeV}$ proton accelerator may produce pairs of heavy leptons with $M_{\ell}$ as large as $10 \mathrm{GeV}$. But, as is also discussed in that section, photoproduction of pairs is not nearly as clean a search method as is the production by $\mathrm{e}^{-} \mathrm{e}^{+}$colliding beams. For this reason, and for many other reasons, much higher energy electronpositron colliding beam facilities are now being discussed. For example, physicists at the Lawrence Berkeley Laboratory and at SIAC are considering $-34-$ 
the design of a $15 \mathrm{GeV}$ electron-positron colliding beam facility called PEP; and Rutherford Laboratory physicists are considering a similar facility called EPIC.

To conclude this section, we note that assuming the existence of an intermediate neutral boson, the $z^{\circ}$, neutral heavy leptons can be produced $^{52}$ through the diagrams in Fig. 13. Here the $\mathrm{E}^{\mathrm{O}}$ is used as an example.

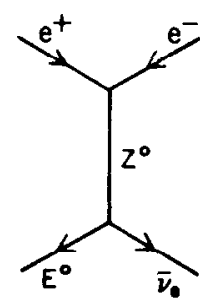

(0)

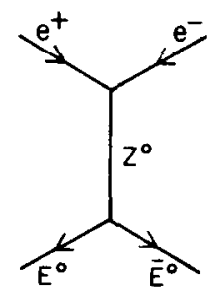

(b)
Fig. 13

Unfortunately, the experimenter finds such searches to be impractical.

Comparing the diagrams in Fig. 13 with the virtual photon production of charged leptons diagram of Fig. 10, it is easy to estimate the change in Eq. 47 --the lepton production cross section. The electromagnetic coupling and photon projection factor $\mathrm{e}^{2} / \mathrm{s}$, where $\mathrm{s}=4 \mathrm{E}^{3}$, is replaced by

$$
\frac{g^{2}}{s+M_{z}^{2}} \sim G, \text { for } M^{2} z^{\circ \gg s}
$$

Thus

the right hand side of Eq. 47 is multiplied by 10

$$
\left(G^{2} / \alpha^{2}\right) E^{4} \sim 10^{-6} E^{4}
$$

Hence

$$
\sigma\left(e^{+} e^{-} \rightarrow E^{o}+\tilde{E}^{\circ} \text { or } E^{o}+\ddot{v}_{e}\right) \sim 10^{-38} \mathrm{E}^{2} \mathrm{~cm}^{2}
$$

where $E$ is in GeV. Even $\mathrm{E}^{\prime} \mathrm{s}$ in the $10-20 \mathrm{GeV}$ range do not yicld sufficient cross section.

C. Photoproduction of Heavy Leptons

1. The Method

For heavy leptons which are Dirac point particles with unit charge, the production cross section for the photoproduction of pairs of these particles can be calculated quite well. 53,54 one of the most recent complete calculation on these processes is that of Kim and Tsai. 53 They give the results of a typical calculation for a beryllium target for $.1 \leq \mathrm{M}_{\ell} \leq 6 \mathrm{GeV}$ and $\mathrm{k}$, the photon energy, up to $200 \mathrm{GeV}$. Figure 14 is a plot of the total cross section for production.

The differential cross section ${ }^{33}$ for the production of one lepton at an angle $\theta$ and momcntum $\mathrm{p}$, summed over all allowed momenta and angles of the other lepton and all hadronic final. status, is

$$
\frac{d \sigma}{d \Omega d p}=\frac{2 \alpha^{3}}{\pi k}\left(\frac{E^{2}}{M_{l}^{4}}\right)\left[\frac{2 x^{2}-2 x+1}{(1+r)^{2}}+\frac{4 x(1-x) r}{(1+r)^{4}}\right] x
$$

Here $\mathrm{k}$ is the photon energy, $\mathrm{M}_{\ell}$ is the lepton mass, $\mathrm{E}$ is lepton's total energy, $\mathrm{x}=\mathrm{E} / \mathrm{k}, \gamma=\mathrm{E} / \mathrm{M}_{\ell}$, and

$$
\mathbf{r}=\gamma^{2} \theta^{2}=E^{2} \theta^{2} / M_{l}^{2}
$$

$x$, defined exactly in Ref. 53 , is a function of the minimum four-momentum transfer to the hadronic vertex and the form f'actors at that vertex. From Eq. (53) we observe the following well-known property of the photoproduction of pairs. When $\theta$ is small so that $\mathbf{r} \ll 1$,

$$
\frac{\mathrm{d} \sigma}{\mathrm{d} s \mathrm{dp}} \sim \frac{1}{\mathrm{M}_{l}^{4}}
$$




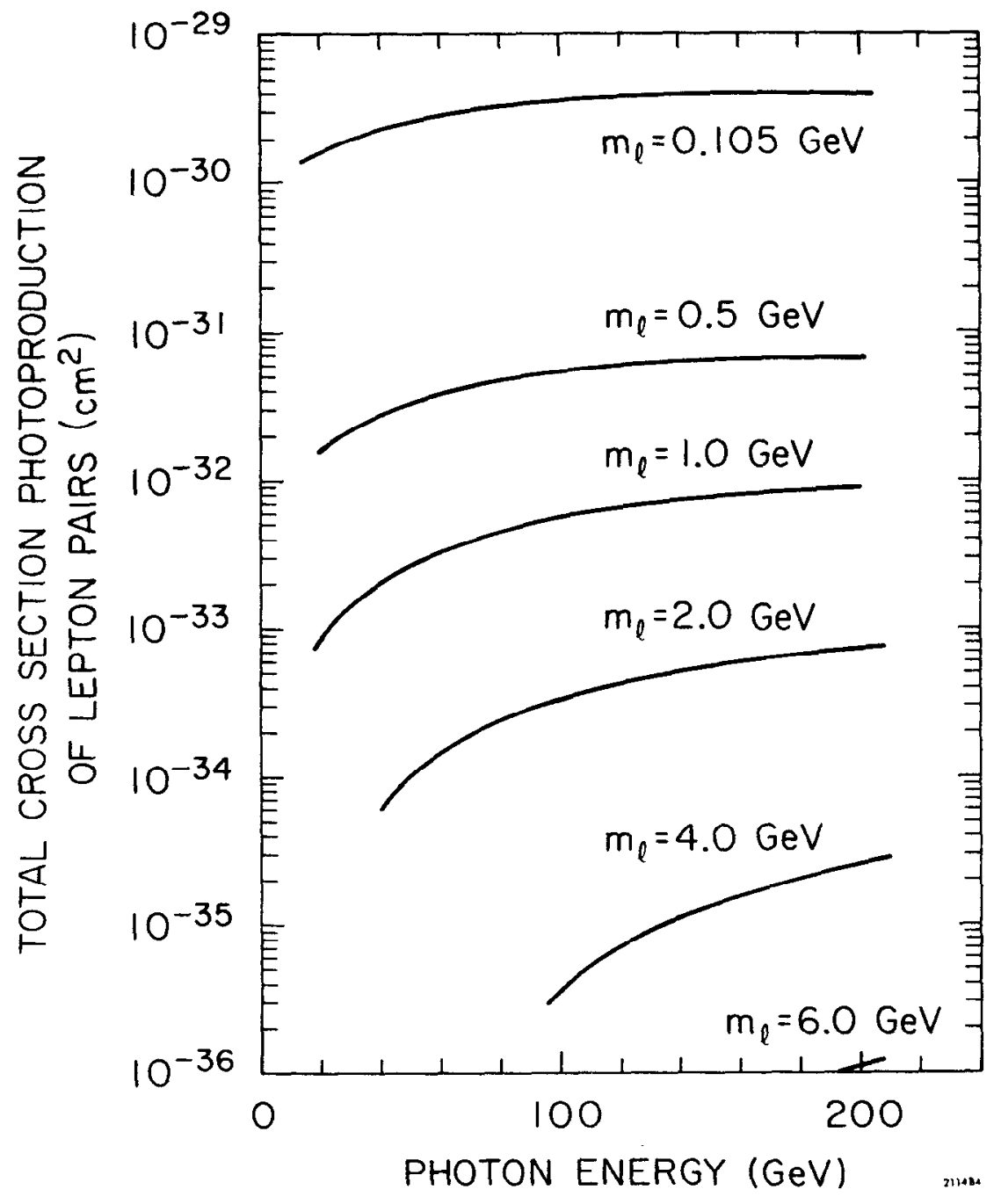

Fig. 14
Thus at small angles the production of high mass heavy leptons is mach suppressed compared to electron or muon praduction. On the other hand, when $\theta E \gtrsim M_{l}$, then $r \gtrsim 1$ and $d \sigma /(d \Omega d p)$ is, except for the $x$ term, independent of $M_{l}$. Hence the crude rule that at large angles, all leptons will be produced in roughly equal numbers.

Separated photon beams need not be used to produce lepton pairs. For example, at electron accelerators, electrons hitting a target of 5 or 10 radiation lengths lead to a double process in the same target, ${ }^{21,55}$ namely

$$
\begin{aligned}
& e^{-}+\text {Nucleus } \rightarrow \mathrm{e}^{-}+\text {Nucleus }+\gamma \\
& \gamma+\text { Nucleus } \rightarrow \ell^{+}+\ell^{-}+\text {hadrons. }
\end{aligned}
$$

At proton accelerators the following sequence of processes can occur in a thick target ${ }^{56}$

$$
\begin{aligned}
& p+\text { Nucleus } \rightarrow \pi^{0}+\text { hadrons, } \\
& \pi^{\circ} \rightarrow \gamma+\gamma, \\
& \gamma+\text { Nucleus } \rightarrow \ell^{+}+\ell^{-}+\text {hadrons. }
\end{aligned}
$$

But the processes of Eq. 57 cannot always be distinguished from other hypothetical processes for making lepton pairs in proton-proton or proton-nucleus collisions. Therefore we postpone further discussions of all proton-proton or proton-nucleus searches to the next section.

Since the relatively high mass heavy leptons under discussion here have very short lifetimes, they must, of course, be detected through their decay modes. Their detection through their hadronic decay modes, if they are heavy sequential leptons or special pair leptons, is very difficult because of the copius direct production of hadrons in photoproduction. The total cross section for the production of hadrons by photons at high energy is about $10^{-28} \mathrm{~cm}^{2}$, 
which is $10^{4}$ to $10^{8}$ times larger than the heavy lepton production cross section in Fig. 14. Therefore past and future searches for heavy sequential leptons almost always rely on the detection of the electrons, the muons, or the neutrinos produced in the leptonic decay modes. For the heavy excited lepton the electron, mion or $y$ must be detected. We now turn to these searches.

2. Past Searches

We know of only one search for short-lived heavy leptons carried out at an electron accelerator. This is a search 55,57 carried out at SIAC in which an $18 \mathrm{GeV}$, high intensity electron beam was used in the production process of Eq. 56 . All charged particles were stopped very quickly by a thick wall of matter. However a heavy lepton with a lifetime shorter than $10^{-10}$ seconds would decay before stopping. The decay mode

$$
\mu^{-} \rightarrow v_{\mu}+\pi^{-}
$$

would yield high energy, $\nu_{\mu}$, neutrinos which would penetrate the wall. 57 Some of these $\nu_{\mu}$, neutrinos might then interact in optical spark chambers, placed downstream of the wall; and these interactions would lead to hadron productions through the production and immediate decay of the $\mu^{*}$. No events which require this explanation were found, and in general no other clear evidence was found for the production of the $H^{\prime}$. But the experiment did not have sufficient sensitivity. 55 And the production, by the processes of Eq. 56 , of heavy sequential leptons with $M_{\mu},>.5 \mathrm{GeV}$ was not excluded.

3. Future Searches

High energy photon beams have been produced at Serpukov and will be produced at FNAL and at the new CERN accelerator. A general summary of proposed FNAL heavy lepton searches by photoproduction, and by other methods, has been given by Heusch and Sandweiss. ${ }^{56}$ As an example, Lee et al ${ }^{58}$ plan to use a photon beam with a maximum energy of $400 \mathrm{GeV}$. The beam hits a 0.1 radiation length beryllium target. Spark chamber hodoscopes, an analyzing magnet, and scintillation and shower counters are used to select and identify e's, $\mu$ 's and $\pi$ 's of greater than $30 \mathrm{GeV} / \mathrm{c}$ momentum produced at between 20 to $50 \mathrm{mrad}$ to the photon beam direction. The trick is to search for those events in which both heavy leptuns have decayed in the decay modes

$$
\begin{aligned}
& \mu^{\prime} \rightarrow \nu_{\mu^{\prime}}+\mu+v_{\mu}, \\
& \mu^{\prime} \rightarrow v_{\mu^{\prime}}+e+v_{e^{\prime}}, \\
& \mu^{\prime} \rightarrow \nu_{\mu^{\prime}}+\pi ;
\end{aligned}
$$

these being the decay modes in which just one charged particle is produced. In particular the experimenters hope to make use of the sharp upper cutoff at $p_{\perp}=M_{\mu}, / 2$ in the transverse momentum $\left(p_{\perp}\right)$ spectrum of the $e, \mu$, or $\pi$.

To give the reader a rough feeling for the sensitivity of such experiments we have shown in Fig. $1^{1}$, the expected photon spectrum for a photon beam of maximum energy $400 \mathrm{GcV}$, produced at a proton accelerator. For a crude calculation of the total rate of production of a heavy lepton of $4.0 \mathrm{GeV} / \mathrm{c}^{2}$ mass we take an average production cross section of $3 \times 10^{-35} \mathrm{~cm}^{2}$ from Fig. 14; and we assume that the accelerator will on the average provide $10^{13}$ protons per pulse and 1000 pulses per hour to the individual experiment. Then in a heavy lepton production target consisting of 1.0 radiation length of beryllium, one would obtain

$$
\frac{\text { total number of mass } 4.0 \mathrm{GeV} / \mathrm{c}^{2} \text { leptons }}{\text { hour }} \sim 100 .
$$

Experiments using small solid angle detectors, such as the ones being described in this section, would appear to have a fractional acceptance for each decay particle from the heavy lepton of about $10^{-1}$ to $10^{-3}$. This includes the angular 


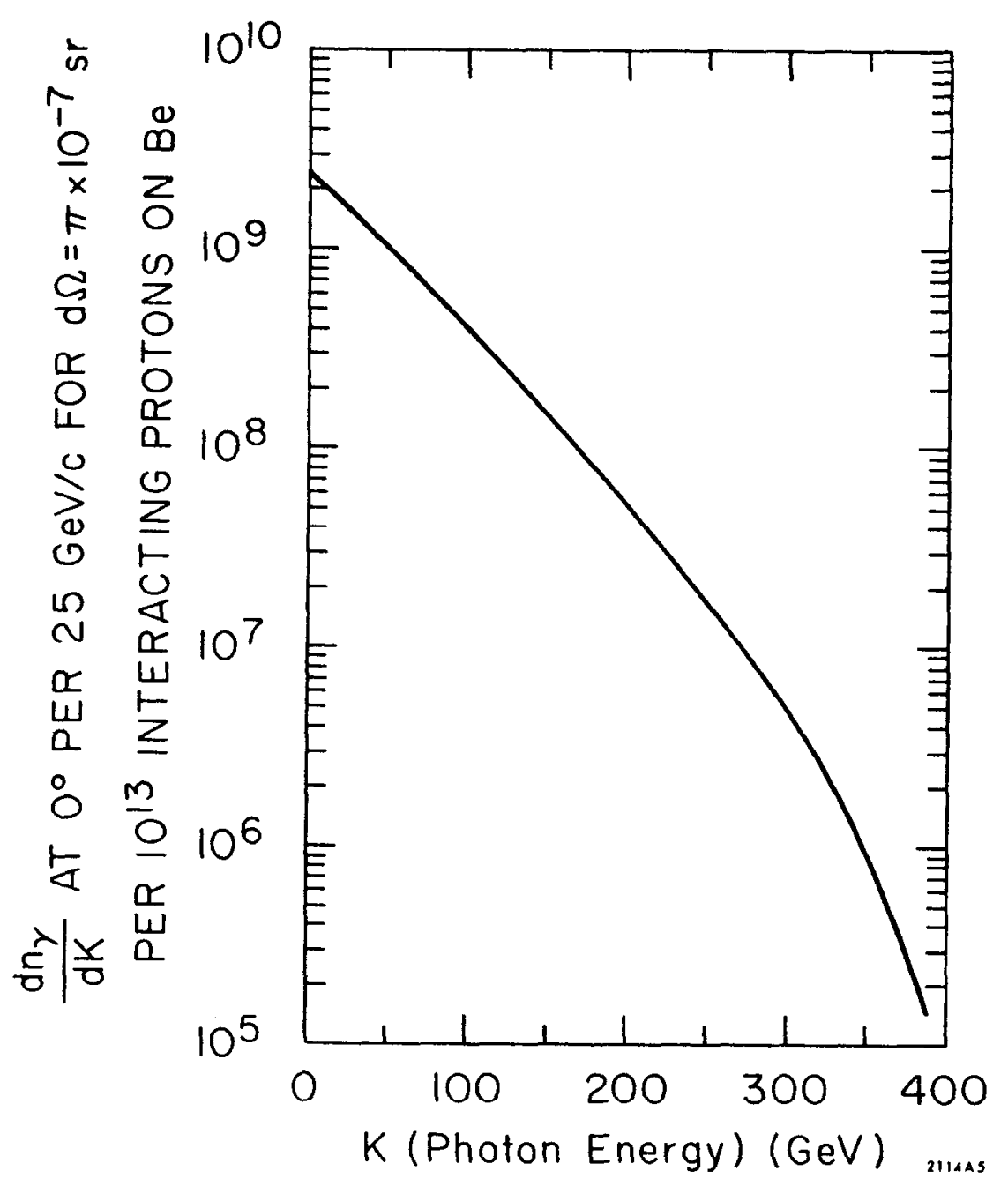

Fig. 15 and momentum acceptance; and the fractional decay into the desired decay mode. Therefore

$$
\frac{\text { detected number of mass } 4.0 \mathrm{GeV} / \mathrm{c}^{2} \text { leptons }}{\text { hour }} \sim 1 \text { to } 10^{-4}
$$

of course higher mass leptons would have even lower detection rates; and to compensate larger solid angle detectors would have to be used.

\section{Heavy Lepton Production in Proton-Proton Collisions}

The interactions of the full intensity primary proton beam of a very high energy proton accelerator with a thick target is the most copious source of secondary particles. And among those particles one might hope to find heavy leptons. Also proton-proton colliding beams facilities, although not as potentially a copious a source of heavy leptons, have the highest upper limit on the mass of the lepton which is kinematically allowed. Therefore proton-proton and proton-nucleus reactions are certainly a good place to look for heavy leptons. However the experimenter is plagued by two severe uncertainties.

(a) The production cross section is not known. We might expect heavy lepton pair production through a timelike virtual photon $\left(\gamma_{\mathrm{v}}\right)$ as shown in Fig. 16. But the

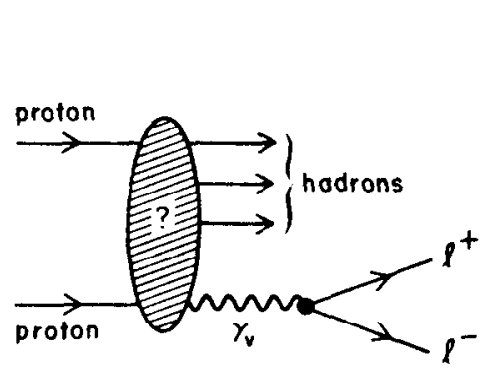

(a)

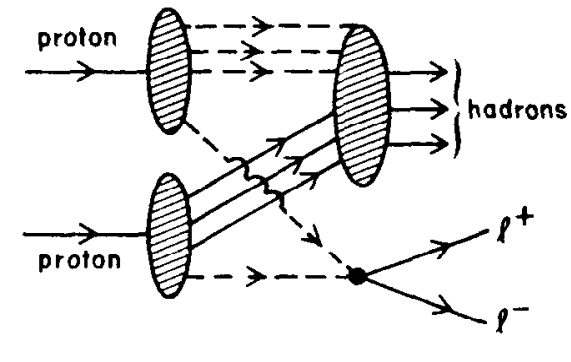

(b)

$2580 \mathrm{AlO}$ 
cross section calculation depends on as yet untested models. An example is provided by the parton model calculations ${ }^{59-61}$ using the diagram in Fig. 160 and the Drell-Yan theory. ${ }^{59}$ In Fig. lób the dashed lines are partons or antipartons, and it is the parton-antiparton annihilation which produces the virtual photon. But st present we don't know if parton model calculations are meaningful in proton-proton collisions.

(b) It is not clear if a recently discovered flux of elections and muons from proton-proton and proton-nucleus collisions is related to heavy lepton production or is another processs which will obscure heavy lepton searches. Briefly, experiminiers ${ }^{62-64}$ at INAL and at the 1 SSR have found that in the afurementioned collisions there are about $10^{-4}$ electrons per pion and about $10^{-4}$ muona per pion at transverse momenta of greater than $1 \mathrm{GeV} / \mathrm{c}$ At present we know very little about the production of these leptons. Are they produced in association with their charged antiparticle or in association with their neutrino? If they belong to charged pairs, are these pairs the same phenomenu seen earlier at lower energies by Christenson et al. ${ }^{65}$ leptons produced by the mechanism in Fig. i6., or do they come from the decay of a more primary particle? Are these primary particles something ordinary like vector mesons - the $\rho^{\circ}$ has the decay $\rho^{\circ} \rightarrow \mu^{+} \mu^{-}$- or are these primary something new - perhaps heavy leptons?

There are a number of reasons to doubt that the primary particles are heavy lepton pairs. The production cross section is very much larger than expected. The same mechanism that produces the heavy lepton pairs, that in Fig. $16 a$ for example, shoula directly produce even more electron pairs and muon pairs. This is because the propagator of $y_{v}$ in Fig. $16 \mathrm{a}$ is $1 / \mathrm{q}^{2}$, and to proluce a pair of mass $\mathrm{M}_{\ell}$ leptons we require $\mathrm{q}^{2}>4 \mathrm{M}_{l}^{2}$.

$$
-43-
$$

However until more is known about these electrons and muons we must reserve judgement as to their origin. We simply don't know if they will be a blessing or a curse to the heavy lepton nunter.

E. Searches for Heavy Excited Leptons Using Charged Lepton-Proton

\section{Elastic Scattering}

1. The Method

If the vertex of Eq. 4 is assumed then the reactions

$$
e+p \rightarrow e^{*}+p, \quad e^{*} \rightarrow e+\gamma
$$

or

$$
\mu^{\prime}+\mathrm{p} \rightarrow \mu^{*}+\mathrm{p}, \quad \mu^{*} \rightarrow \mu+\gamma
$$

can occur. ${ }^{66}$ The cross section depends of course on $\lambda^{2}$. The reaction can be identified by looking for a sharp peak other than the elastic peak in the momentum spectrum of the recoil proton. Therefore this search method does not require any knowledge of the nature of the $e^{*}$ or $\mu^{*}$, it only requires the assumption of an electromagnetic coupling of the e or $\mu$ to the heavy lepton.

2. Past Searches

Experimenter at Orsay, 67 DESY, ${ }^{68}$ and $\mathrm{CEA}^{69,70}$ have used this method to search for $e^{*}$ heavy excited leptons. But none have been found. Figure 17 a summarizes the limits on $\lambda$ set by these searches. Also shown in Fig. 170 are the $\lambda$ limits found in the colliding beam searches 47,48 for the $e^{*}$ discussed in Sec. V. B2.

H. Gittleson et al.,$^{71}$ at the Brookhaven AGS used the reaction in Eq. $60 \mathrm{~b}$ to look for the $\mu^{*}$. No $\mu^{*}$ was found in a mass search range of 0 to $2 \mathrm{GeV} / \mathrm{c}^{2}$; the limits on $\lambda$ are given in Fig. 17 and to quote these authors 71 "This (experimental result) would appear to rule out the Ramm particle (discussed in Sec. IV.A2.)" The g-2 limits on $\lambda$ in Fig. $l_{i}^{7}$ come from the measurement discussed in Sec. VI. B. 


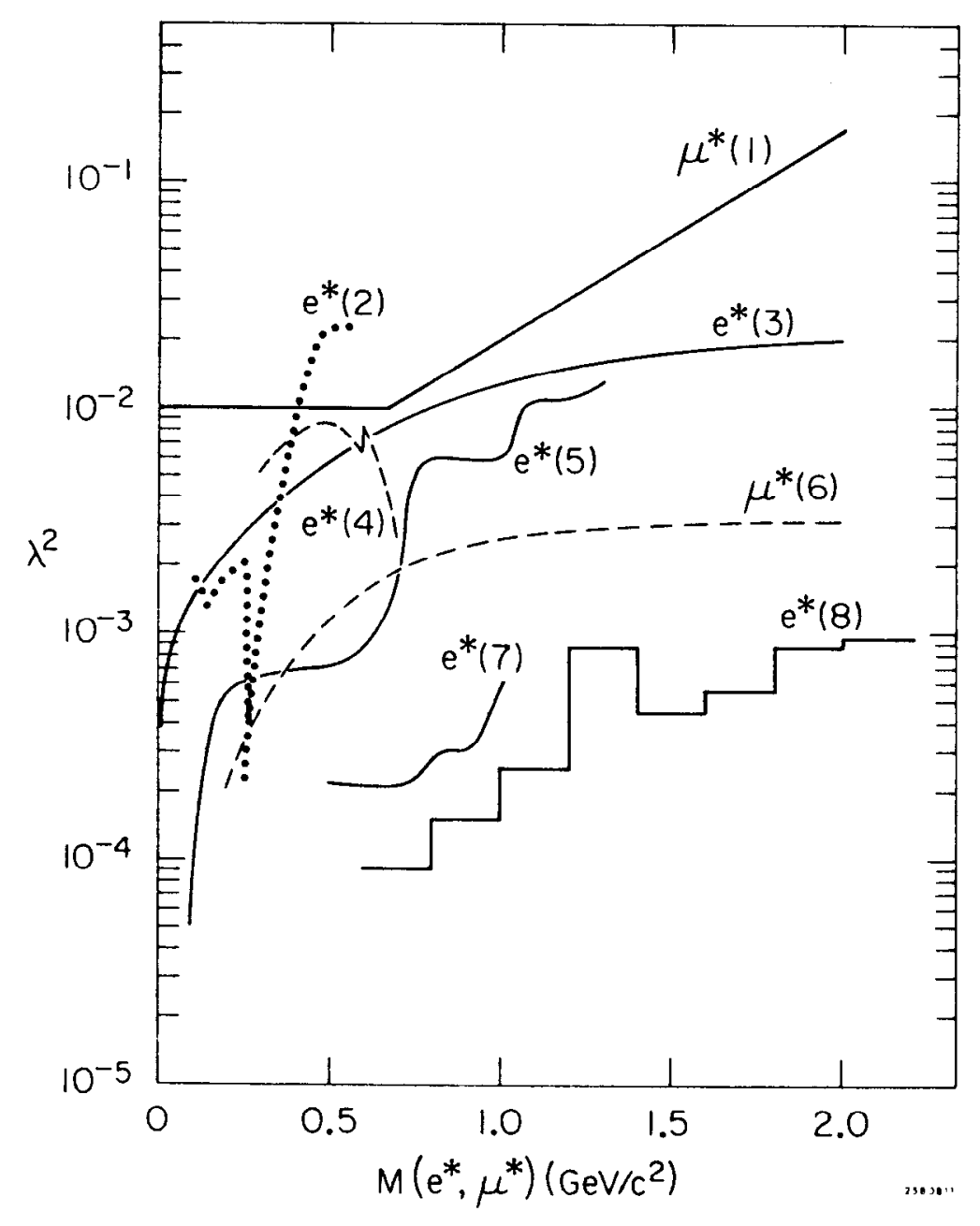

Fig. 17. Comparison of upper limits on the value of $\lambda^{2}$, Eq. 4, for the $e^{*}$ and i1*. References are: 1 . H. Gittleson et al., Phys. Rev D., to be published, ( $\mu-p$ scattering); 2. C. Betourne et al., Phys. Letters 17, 70(1065), (e-p scattering): 3. A. De Rujuia and $B$. Lnutrup, Ietter Nuovo Cimento 3,49 (1972), (g-2 of electron); 4. R. Budnitz et al., Phys. Rev. 141, 1313(1966), (e-p scattering) 5. C.D. Boley et al., Phys. Rev. 167, 1275 (1968), (e-p scattering); 6. A. De Rújula and B. Lautrup, Let tere Nuovo Cimento 3, 49 (1972), (g-2 of muon); 7. H.J. Behrend et al., Phys. Rev. Letters 15, 900 (1.65), (e-p scattering); 8. C. Bacci et al. , Phys. Letters $44 \mathrm{~B}$, (1265), (e-p scattering); 8. C. Bacci et al., Phys. Letters $44 \mathrm{~B}$,
$530(1973),(e+e \rightarrow e+e+y)$.

\section{Future Searches}

Higher energy lepton beams are now available at the new higher energy accelerators. For a primary lepton beam of energy $E$ this search method extends to excited lepton masses $M_{l_{*}} \approx \sqrt{2 E M_{p}}$. However, one should remember that the square of the minimum four-momentum-transfer $\left|q^{2}\right|_{m i n}$ goes as

$$
q^{2} \min =\frac{M_{l_{*}}^{4}}{4 E^{2}} .
$$

The form factors at the proton vertex will lead to a rapid loss of sensitivity as $\left|q^{2}\right|_{\min }$ increases.

F. Searches for Heavy Excited Leptons in Lepton Bremsstrahlung

1. The Method

This search method, which is closely related to the method discussed in the last section, consists simply of the study of the invariant mass $M_{e r}$ in the bremsstrahlung process

$$
\mathrm{e}+\text { nucleus } \rightarrow \mathrm{e}+\gamma+\text { mucleus. }
$$

An exactly similar method can be used to search for the $\mu^{*}$ in muon bremsstrahlung.

2. Past Searches

No evidence for the $e^{*}$ has been found in studies of electron brcmsstrahlung. $32,72,73$ The most extensive study covers the mass range of . I to $1.2 \mathrm{GeV} / \mathrm{c}^{2}$. But as pointed out by Lichtenstein, ${ }^{32}$ these bremsstrahlung experiments are quite a bit less sensitive for heavy lepton searches compared to the lepton-proton scattering experiments previously descirbed.

3. Future Searches

A very high energy search for the $e^{*}$ in electron bremsstrahlung has been proposed by J.F. Crawford et al. ${ }^{74}$ This experiment, to be carried out at FNAL, is designed for electron beams of several hundred $\mathrm{GeV}$ energy. 
G. Searches for Heavy Leptons Using Charged Lepton-Proton Inelastic Scattering

Charged lepton-proton inelastic scattering can be used to search for heavy

leptons. However we postpone the discussion of this search method to Sec. $X$

where we discuss lepton-proton inelastic scattering in detail.

H. Searches for Special Pair Leptons Using Neutrino-Nucleon Inelastic Scattering

The availability of high energy, high intensity neutrino beams at the new proton accelerators, and the interest in theories uniting weak and electromagnetic which may require heavy leptons (Sec. II.C) have stimulated searches for the $\mathrm{E}^{+}, \mathrm{E}^{\mathrm{O}}$ and $\mathrm{M}^{+}, \mathrm{M}^{\mathrm{O}}$ members of special lepton pairs. Recalling from Sec. II.C that the $M^{+}, M^{\circ}$ are assigned the lepton numbers of the $\mu^{-}, \nu_{\mu}$, we see that the $\mathrm{M}^{+}$or $\mathrm{M}^{\circ}$ can be produced throukh

$$
\begin{aligned}
& \nu_{\mu}+\text { nucleon } \rightarrow M^{+}+\text {hadrons } \\
& v_{\mu}+\text { nucleon } \rightarrow M^{\circ}+\text { hadrons }
\end{aligned}
$$

The $\mathrm{M}^{+}$or $\mathrm{M}^{0}$ are to be detected through their decay products. For example, the decay $\mathrm{M}^{+} \rightarrow \nu_{\mu}+\mu^{+}+\nu_{\mu}$ allows the two-step process

$$
v_{\mu}+\text { nucleon } \rightarrow M^{+}+\text {hadrons } \rightarrow \mu^{+}+2 v_{\mu}+\text { hadrons }
$$

Here a $\mu^{+}$is produced in contrast to the usual one-step inelastic process

$$
v_{\mu}+\text { nucleon } \rightarrow \mu^{-}+\text {hadrons }
$$

where $\mu^{-}$is produced. A thorough discussion of the various search methods including those for the $E^{\circ}$ and $M^{\circ}$ has been given by Cline. ${ }^{75}$

There are three advantages of this method, assuming that the weak interaction coupling constants of the $M$ and $E$ are not to different from that of the $e$. First, the production cross section can be estimated. Second the desired signal is not hopelessly buried in background as it may be in proton-proton production of heavy leptons (Sec. V.D). Third, the probably dominant multi-hadron decay modes, such as Eq. 37, can be used if the two-step process

$$
\nu_{\mu}+\text { nucleon } \rightarrow M^{+}+\text {hadrons } \rightarrow \nu_{\mu}+\text { hadrons }
$$

can be distinguished from the one-step neutral current interaction

$$
\nu_{\mu}+\text { nucleon } \rightarrow \nu_{\mu}+\text { hadrons }
$$

The disadvantage of the method is that it is restricted to looking for spectal pair leptons which have the lepton numbers of the e or $\mu$.

No such heavy leptons have been found. For examplc, Barish et al. ${ }^{76}$ searched for the $\mathrm{M}^{+}$up to masses of $10 \mathrm{GeV} / \mathrm{c}^{2}$ using $v_{\mu}$ neutrinos at FNAL. The sensitivity, as we expect, depends upon what one assumes for the $M^{+}$ coupling constant and $R_{\text {decay }}$ (Eq. 23). 
VI. COMPARISON OF SOME STATIC PROPERTIFS OF THE MUON AND THE ELECTRON

In this section we compare some of the static properties of the muon and the electron, properties which are particulaxly relevent to later discussions. We adopt the point of view that the property of the electron is the standard and that the corresponding property of the muon requires comment.

\section{A. Electric Charge}

Four properties of the man - the electric charge $e_{\mu}$, the mass $m_{\mu}$, the magnetic moment $\mu_{\mu}$ and the gyromagnetic ratio $g_{\mu}$ - are connected by the relation

$$
\mu_{\mu}=\left(\frac{g_{\mu}}{2}\right)\left(\frac{e_{\mu} h}{2 m_{\mu} c}\right)
$$

$g_{\mu}$ and $\mu_{\mu}$ have been determined with great precision; ${ }^{1,4,77} .3$ parts per million and 12 parts per million respectively. Therefore $e_{\mu}$ can be determined if $m_{\mu}$ is known from an independent measurement. Such a measurement is provided by the study of the mu-mesic atom, an atom in which a negative mon is captured in an atomic orbit. ${ }^{78}$ Ignoring relativistic corrections, fine structure and hyperfine structure, the $\mathrm{n}^{\text {th }}$ energy level of such an atom is given by the Bohr formula

$$
E_{n}=\frac{-m_{\mu} e_{\mu}^{2}\left(z_{p}\right)^{2}}{2 n^{2} \hbar^{2}}
$$

We have distinguished the muon charge $\mathrm{e}_{\mu}$ from the charge on the nucleus Ze $\mathrm{p}^{\text {. }}$ By measuring the energy difference between levels, $m_{\mu}$ or more precisly the combination $m_{\mu} e_{\mu}^{2}$ can be determined. This measured value of $m_{\mu} e_{\mu}^{2}$ combined with Eq. 67 and the known values of $\mu_{\mu}, \mathrm{g}_{\mu}, \hbar, c$ and $\mathrm{e}_{\mathrm{e}}$ (the charge on the electron) yields ${ }^{4}$

$$
e_{\mu} / e_{e}=1 \pm\left(4 \times 10^{-5}\right)
$$

But a much lower limit can be obtained ${ }^{79}$ by observing that if charge is conserved in the mon decay process

$$
\mu \rightarrow e+v_{\mu}+\bar{v}_{e}
$$

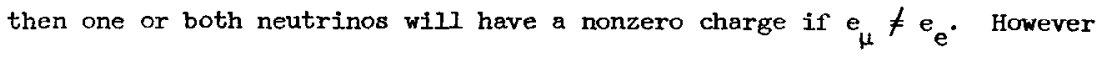
neutrinos could then be pair produced by low energy photons, leading to an additional mechanism for energy loss in stars! Astrophysical considerations then set an upper limit on the charge that could be possessed by a neutrino. This limft leads to the conclusion that

$$
e_{\mu} / e_{e}=1 \pm 1 \times 10^{-13}
$$

\section{B. Gyromagnetic Ratio}

The gyromagnetic ratio, $\mathrm{B}_{\mu}$, can be calculated exactly from quantum electrodynamics, once the muon mass is known, if strong interactions are ignored. (Fortunately the influence of the strong interactions on $G_{\mu}$ is small; we will give the estimated size of the effect below.) The Dirac relativistic theory of the electron or the muon yields $g=2$. The Feynman diagram for the interaction of a muon with an external magnetic field (which yields $\mathrm{g}=2$ ) is given in Fig. 18

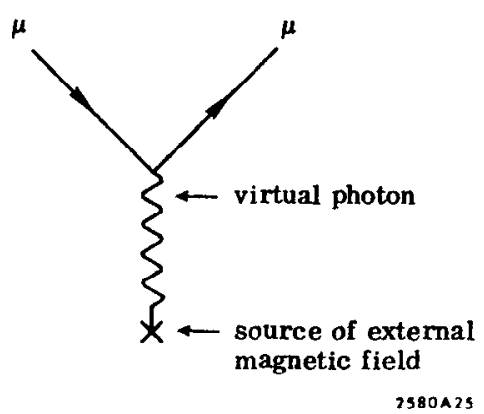

Fig. 18 
But quantum electrodymamics shows that there is an anomalous magnetic moment so that $g$ is not exactly 2. It is conventional to set

$$
\left(g_{\mu}-2\right) / 2=a_{\mu}=\frac{1}{2}\left(\frac{\alpha}{\pi}\right)+A_{2}\left(\frac{\alpha}{\pi}\right)^{2}+A_{3}\left(\frac{\alpha}{\pi}\right)^{3}+\ldots
$$

The coefficients $A_{i}$ are all of the order of magnitude of 10 or less so that $\alpha_{\mu}$ is very small. Nevertheless it has been measured to great accuracy. The measurement of $a_{\mu}$ is a measurement of the combined effect of terms like those in Fig. 19.
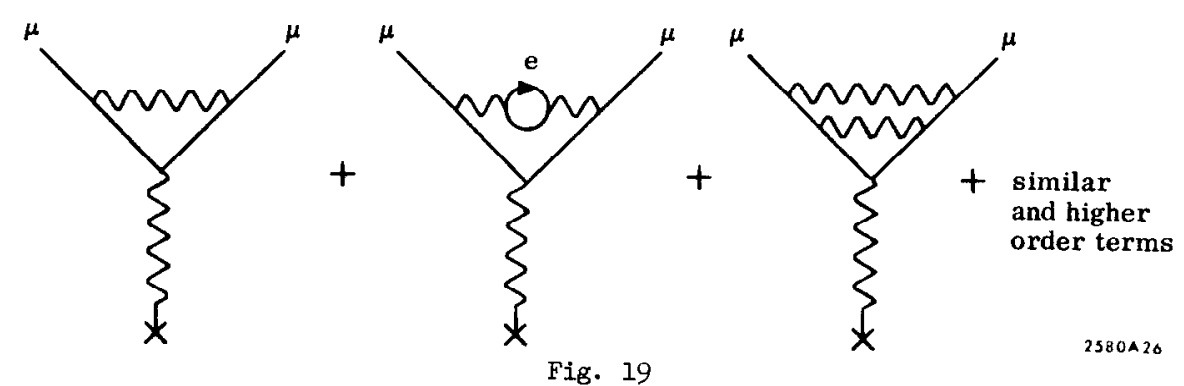

The most recent results of Farley, Picasso and their colleagues ${ }^{1}$ at CEkN yield

$$
a_{\mu}^{e x p}=(116616 \pm 31) \times 10^{-8}
$$

and quantum electrodynamics yields ${ }^{2,77}$

$$
a_{\mu}^{\text {theory }}=(116588 \pm 2) \times 10^{-8}
$$

Thus experiment and theory are in agreement. Even more precise agreement is found for the electron. 77

$$
\begin{aligned}
& a_{e}^{e x p}=\left(\begin{array}{lll}
1 & 159657.7 \pm 3.5
\end{array}\right) \times 10^{-9} \\
& a_{e}^{\text {theory }}=\left(\begin{array}{lll}
1 & 159655.3 \pm 2.5
\end{array}\right) \times 10^{-9}
\end{aligned}
$$

Therefore with respect to the measurement of $\mathrm{g}-2$, once the mass of the muon is taken into account, there is not observable difference between the muon and the electron.

Incidently, if the $M^{\circ}$ special pair lepton exists, the diagram in Fig. 20a will contribute to $g_{\mu}$, where $W$ is the hypothetical charged

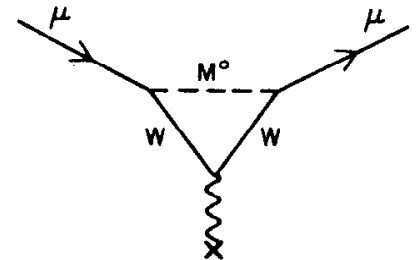

(a)

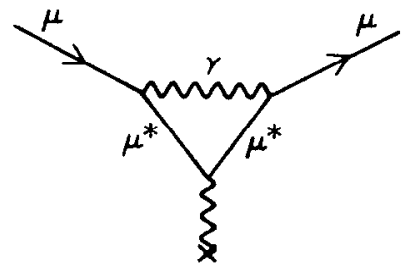

(b) 25801415
Fig. 20

intermediate boson. ${ }^{16}$ Therefore the comparison of $\mathrm{g}_{\mu}^{\exp }$ with $\mathrm{g}_{\mu}^{\text {theory }}$ limits the contribution of this diagram. 19 Unfortunately the sensitivity as a function of $\mathrm{M}^{\circ}$ mass also depends upon assumptions about the $\mathrm{W}$ mass and the relevent weak interaction coupling constants. ${ }^{19}$ No useful limits on the $\mathrm{M}^{\circ}$ 's existence can be set as yet by this method. A similar remark applies to searching for the $\mathrm{E}^{\circ}$ by using $\mathrm{g}_{\mathrm{e}}$ comparisons.

Turning next to Fig. $18 \mathrm{~b}$ we see that the existence of a $\mu^{*}$ can contribute to $g_{\mu}-2$. Similarly there is an $e^{*}$ contribution to $g_{e}-2$. The limits on the corresponding $\lambda^{\prime}$ 's of Eq. 4 are given in Fig. 17. Obviously there is no evidence for an $e^{*}$ or $\mu^{*}$ from Eyromagnetic ratio measurements. 80 


\section{MU-MFS IC ATOMS}

If the muon is a point Dirac particle the energy levels of the mu-mesic atom $^{78}$ will be given by Eq. 68 corrected for fine structure, hyperfine structure, relativistic effects, quantum electrodynamic effects, nuclear size and nuclear charge distribution. All but the nuclear corrections can be calculated from known and accepted theory. The corrections for the size and charge distribution of the nucleus must be determined by experiment. In fact the major purpose of mu-mesic atom experiments is to measure those properties of the nucleus.

By measuring many mu-mesic X-ray lines from a mu-mesic atom, a large amount of interrelated information on the spacing of the energy levels is obtained. Some of this information, particularly that coming from lower energy levels, can be used to derive the relevant nuclear properties. These derived nuclear properties can then be used to calculate the nuclear corrections in the higher energy levels, where those corrections are relatively small. In this way one can attempt a self-consistent calculation of all the mu-mesic X-ray lines. If such a self-consistent calculation cannot be made, the usual hypothesis is that the theory of atomic energy levels contains an error or that the theory of how to derive and correct for the nuclear properties contains an error. If nefther of these errors could be found, then one would have to assume that the problem lay with the muon. The muon might not be a point particle or the muon might have an anomalous interaction with the nucleus. Thus high precision measurements of the $\mathrm{X}$-ray lines from mu-mesic atoms provide a test of muon-electron universality.

The search for anomalous effects will be most sensitive if the distance between the nucleus and the mon is relatively small. For if the mon is not a point particle, this will be most evident for small distances. Also any anomalous muon-nucleus interaction is likely to fall off rapidly with distance if, as discussed in Secs. IX, $X$, and XI, the interaction is strongest at large four-momentum transfers. Thus the search for anomalous effects in mu-mesic atoms is best carried out with high- $Z$ atoms such as lead.

Three high precision measurements of X-rays from high- $Z$ ma-mesic atoms have been made. ${ }^{81}$ Two of these experiments, Dixit et aㅣ., and Walter et aI., find small discrepancies from theoretical predictions ${ }^{82}$ - the measured energy difference between two atomic levels being smaller than the theoretical prediction. The discrepancies are very small; for example, about $70 \mathrm{ev}$ for the 438,000 ev energy difference between the $5 \mathrm{~g}_{7 / 2}$ and $4 f_{5 / 2}$ levels in lead. And the discrepancies are only about 3 standard deviations. A thorough review of the relation of these discrepancies to anomalous muon-hadron interactions has been given by okun and zakharov. $^{83}$ We don't know how to interpret these possible discrepancies and shall not comment further on them, but this does not mean that they should be ignored. 
VIIT. HIGH ENERGY REACTIONS OF MUONS AND ELECTRONS

Although the static and atomic properties of the charged leptons show no unexplained differences, one might hope that differences will appear when the dynamic properties of the charged leptons are measured at high energy. For high energies were required to reveal the richness and complexities of the strong interactions. Might not high energies also reveal unsuspected complexities in muon and electron physics? The high energy reactions of the charged leptons may be divided into three classes.

1. One class consists of those reactions in which a neutrino is absorbed or produced. Those reactions as presently measured show no violation of muonelectron universality. ${ }^{3,84}$ But the high energy experiments ${ }^{84}$ in this class only have precisions of the order of 10 or 20 percent. Therefore we have not yet had stringent tests of mon-electron universality in this class of reactions.

2. Another class consists of purely electromagnetic reactions in which no hadron participates or in which the hadron has only an auxiliary role acting as an almost static source of electric charge. Examples are the colliding beam reactions

$$
\begin{aligned}
& e^{+}+e^{-} \rightarrow e^{+}+e^{-}, \\
& e^{+}+e^{-} \rightarrow \mu^{+}+\mu^{-} ;
\end{aligned}
$$

and muon bremsstrahlung

$$
\mu+\mathrm{p} \rightarrow \mu+\mathrm{p}+\gamma
$$

with very small momentum transfer to the proton. Many of these experiments have been reviewed ${ }^{2}$ with respect to tests of quantum electrodynamics. Some have also been reviewed at this conference by M.J. Tannenbaum ${ }^{85}$ with respect to the search for mon-electron differences. In particular he discussed a recent experiment ${ }^{86}$ confirming that muons, like electrons, obey Fermi-Dirac statistics. Except for some early experiments, all experiments in this class confirm that the charged leptons are point Dirac particles obeying quantum electrodynamics. Thus all these experiments confirm electron-muon universality in purely electromagnetic reactions. We will review in sec. IX some of the electron-positron colliding beam experiments which are relevant to the mejor concorns of this paper.

3. The third class of reactions, those which we shall emphasize in the remainder of this article, consist of reactions in which hadrons play an intimate role. Our interest in this class of reactions has two origins. First, as we shall discuss later, these ractions provide a way to search for spatial structure in the charged leptons; a way to test if the charged leptons are truly point Dirac particles. (Some Class 2 reactions also test for spatial structure.) Second, a speculation which particularly intrigues us is that the leptons may in some very reduced manner take part directly in the strong interactions. After all, the mass difference between the mon and the electron is almost a pion mass and thus could be caused by the strong interactions. To see if the charged leptons in any way directly take part in the strong interactions, it is desirable to have hadrons present. - hadrons act as a source for the strong interactions.

In the interaction of muons (or electrons) with protons we can consider two kinds of processes; elastic scattering where

$$
\mu+p \rightarrow \mu+p
$$

and inelastic scattering where

$$
\mu+p \rightarrow \mu+\text { (any set of } 2 \text { or more hadrons) }
$$

Examples of inelastic scattering are:

$$
\begin{aligned}
& \mu+p \rightarrow \mu+p+\pi^{0} \\
& \mu+p \rightarrow \mu+n+\pi^{+}+\pi^{0} \\
& \mu+p \rightarrow \mu+\Sigma^{0}+K^{+} \\
&-56-
\end{aligned}
$$


In these clastic or inelastic scattcring reactions, the cherged lepton is not altered in the reaction. This distinguishes these processes from neutrino induced reactions of Class 1 .

\section{TX. MUON-PROTON EIASTIC SCATTERING AND FORM FACTORS}

A. Theoretical Background

We will consider first elastic scattering, and to set the stage we will discuss electron-proton elastic scattering. To a precision of a few percent all data on electron-proton elastic scattering is explained by the Feymman alagram in Fig. 21.
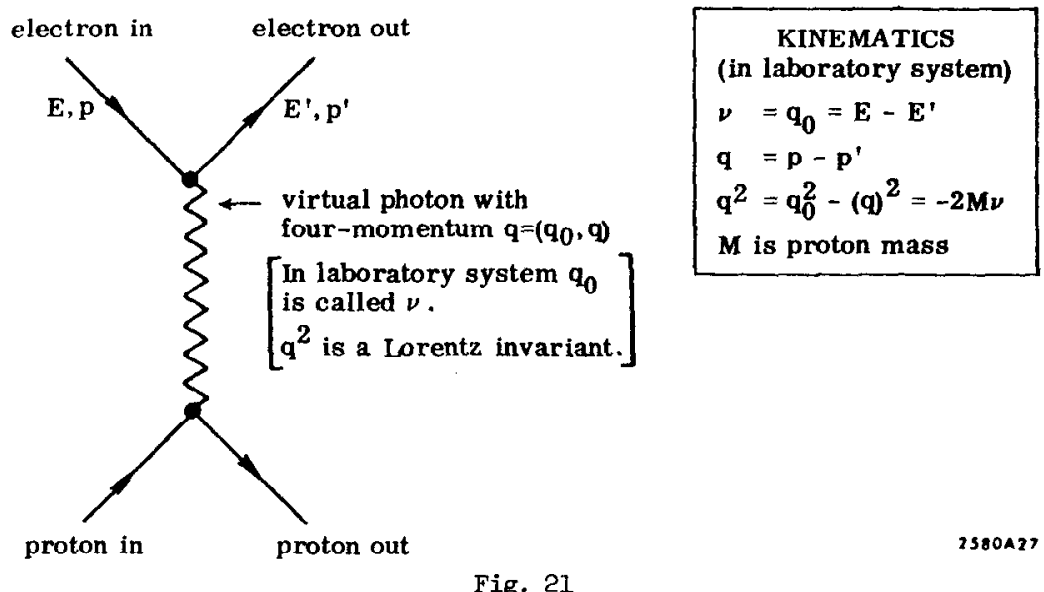

in which only one photon is exchanged. All experiments agree 87 that the differential cross section for this elestic scattering process is described by the equation

$$
\left(\frac{d \sigma}{d q^{2}}\right)_{e p, e l a s}=\left(\frac{d^{\sigma}}{d q^{2}}\right)_{\mathbb{N S}}\left[\frac{G_{E}^{2}\left(q^{2}\right)+\pi_{M}^{2}\left(q^{2}\right)}{1+T}+2 \pi G_{M}^{2}\left(q^{2}\right) \tan ^{2} \frac{\theta}{2}\right]
$$

This equation, the Rosenbluth rormula, assumes that the electron is a point Dirac particlc with only electromagnetic and wcak interactions. The equation is written for scattering in the lahoratory system, $\theta$ is the electron scattering angle and 
$\tau=\left|q^{2}\right| / 4 M^{2} \cdot\left(d \sigma / d q^{2}\right)_{N S}$ is the differential cross section for the scattering of an electron by a spin-zero point proton; NS denotes no spin. $\left(\mathrm{d} \sigma / \mathrm{dq}^{2}\right)_{\mathbb{N S}}$ is a function only of the total energy of the system and $\theta$; it is completely specified by quantum electrodynamics. $\mathrm{G}_{\mathrm{E}}\left(\mathrm{q}^{2}\right)$ and $\mathrm{G}_{\mathrm{M}}\left(\mathrm{q}^{2}\right)$ are the proton form factors.

They take into account that the proton has nonzero spatial extent, and that the proton has strong interactions. If the proton were a point Dirac lepton $G_{F}$ and $G_{M}$ would both equal unity for all values of $q^{2}$. The crucial variable is $q^{2}$, the square of the four-momentum transferred from the lepton vertex. $q^{2}$ is always spacelike in this process and in our metric is negative. In this article energy units will always be GeV, momentum units will be GeV/c and the units of $q^{2}$ will be $(\mathrm{GeV} / \mathrm{c})^{2}$. Also unless $\mathrm{h}$ and $\mathrm{c}$ appear explicitly in a formula, they have both been set equal to 1 . We remind you that it is found experimentally ${ }^{87}$ that

and

$$
\mathrm{G}_{\mathrm{E}}\left(\mathrm{q}^{2}\right) \approx 1 /\left[1+\left|\mathrm{q}^{2}\right| / \cdot 71\right]^{2}
$$

$$
G_{M}\left(q^{2}\right) \approx 2.79 G_{E}\left(q^{2}\right)
$$

$\mathrm{G}_{\mathrm{E}}$ and $\mathrm{G}_{\mathrm{M}}$ are functions of $\mathrm{q}^{2}$ which is a Lorent $z$ scalar. Thus they express in a relativistically correct way the efrects of the hadronic and non-pointlike nature of the proton. When $\left|q^{2}\right|$ is small compared to $M^{2}$, we can treat the proton nonrelativistically and provide a simple physical picture of the meaning of these G's. ${ }^{88,89}$ For $\left|q^{2}\right| \ll M^{2},\left|q^{2}\right| \approx|q|^{2}$ where $q$ is the three-momentum transferred to the proton. Then $G_{E}\left(q^{2}\right) \approx G_{E}\left(|q|^{2}\right)=G_{E}(q)$ where $G_{E}(q)$ is the three-dimensional Fourier transform of the electric distribution.

Explicitly

$$
G_{E}\left(q^{2}\right) \approx G_{E}(q)=\int \rho_{E}(r) e^{i q} \cdot r d^{3} r
$$

$\rho_{\mathrm{E}}(r)$ is the charge density distribution and is normalized by

$$
\int \rho_{E}(r) d^{3} r=1
$$

$\mathrm{G}_{M}\left(\mathrm{q}^{2}\right)$ can be similarly interpreted. If the proton is contained within a sphere of $R$, then for $|q| R \ll 1$

$$
G_{E}(q)=1-\left(\frac{1}{6}\right)|q|^{2}\left\langle r^{2}\right\rangle_{E}+\left(\frac{1}{120}\right)|q|^{4}\left\langle r^{4}\right\rangle_{E}+\cdots
$$

Here $\left\langle r^{2}\right\rangle_{E}$ and $\left\langle r^{4}\right\rangle_{E}$ are the average values of $r^{2}$ and $r^{4}$ respectively over the charge distribution of the proton. It is also possible to expand the furction $G_{E}\left(q^{2}\right)$ in the relativistically invariant form

$$
G_{E}\left(q^{2}\right)=1+a_{1} q^{2}+a_{2}\left(q^{2}\right)^{2}+\cdots
$$

But the coefficients in Fq. $74 \mathrm{c}$ can be rigorously assigned ${ }^{89}$ their corresponding meanings in Eq. $74 b$ only if $\left|q^{2}\right| \ll M^{2}$.

Now if the muon is a pure Dirac point particle we can use Eq. 71 for monproton elastic scattering. There are small effects due to the muon mass, which we have not exhibited explicitly; but these are known. Then

$$
\left(\frac{d \sigma}{d q^{2}}\right)_{\mu p, e l a s}=\left(\frac{d \sigma}{d q^{2}}\right)_{e p, e l a s}
$$

But suppose the muon is not a point particle; suppose the muon, like the proton, has a form factor $G_{\mu}\left(q^{2}\right)$. Then Eq. 75 becomes

$$
\left(\frac{d \sigma}{d q^{2}}\right)_{\mu p, e l a s}=\left(\frac{d \sigma}{d q}\right)_{e p, e l a s} G_{\mu}^{2}\left(q^{2}\right)
$$

of course the most general modification ${ }^{90}$ of Eq. 75 would require the introduction of two form factors corresponding to $G_{E}$ and $G_{M^{\prime}}$ But our very primitive knowledge of the structure of the rmon does not warrant such a refinement. We 
have no theoretical guidance to what $G_{\mu}\left(q^{2}\right)$ might be. But the data reviewed in Sec. VI show that with great precision the static properties of the muon are those of a point Dirac particle. Therefore at $q^{2}=0$ we must have $G_{\mu}(0)=1$. comentionally we take a form analogous to the proton form factor and write

$$
G_{\mu}\left(q^{2}\right)=1 /\left[1-q^{2} / \Lambda_{\mu}^{2}\right]
$$

When $q^{2}$ is spacelike, and hence negative in our metric, we write Eq. (77a) in the form

$$
G_{\mu}\left(q^{2}\right)=1 /\left[1+\left|q^{2}\right| / \Lambda_{\mu}^{2}\right] \text {. }
$$

Note however that unlike Eq. $7_{2}$, only the first power $\left[1+\left|q^{2}\right| / \Lambda_{\mu}^{2}\right]$ appears in the denominator. $\Lambda_{\mu}$ is a sort of inverse measure of the deviation of the muon from a point particle. The smaller $\Lambda_{\mu}$, the greater the deviation. The form of Eq. 77 is actually not as restrictive as it might appear to be. As we sholl see later in this paper, all experiments have led to values of $\Lambda_{\mu}^{2}$ which are much larger than the $\left|q^{2}\right|$ values occurring in the experiment. Therefore Eq. (77v) is well approximated by

$$
G_{\mu}\left(q^{2}\right) \approx 1-\left|q^{2}\right| / n_{\mu}^{2}
$$

Therefore we are actually allowing $G_{\mu}\left(q^{2}\right)$ to differ from 1 by a term linear in $\left|q^{2}\right|$; this is certainly a simple enough assumption.

Comparing Eq. $77 \mathrm{c}$ with Eq. $74 \mathrm{~b}$ we are tempted to make the identification $\langle r\rangle_{\mu} / 6=1 / \Lambda_{\mu}^{2}$

or

$$
\sqrt{\left\langle r^{2}\right\rangle_{\mu}}=\sqrt{6 / \Lambda_{\mu}^{2}}=\left(.48 / \Lambda_{\mu}\right) \times 10^{-13} \mathrm{~cm}
$$

where $\Lambda_{\mu}$ is in $\mathrm{GeV} / \mathrm{c}$. But this identification is only rigorous 89 if $\left|q^{2}\right| \ll\left(\text { mass }_{\mu}\right)^{2}$. We shall see that in the high energy experiments $\left|q^{2}\right| \gg$ (muon mass) ${ }^{2}$. Therefore for these experiments we shall have to be cautious in our interpretation of $1 / \Lambda_{\mu}$ as an indication of the size of the mon.

But a mon might differ in other ways from an electron. There might be a special particle, the $x$ particle, that couples to muons and hadrons but not to electrons. Then muon-proton elastic scattering would be the result of two axplitudes whose diagrams are given in Fig. 22
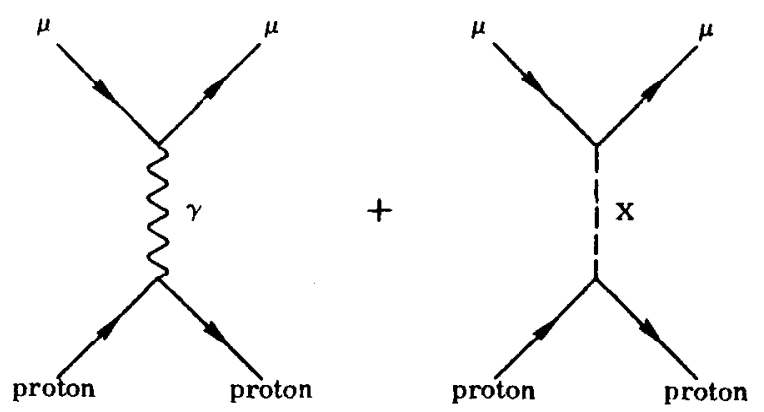

25BOA28

Fig. 22

This would produce some deviation from Eq. 75 , but the nature of the deviation cannot be determined because we do not know what $X$ is. Therefore we continue to use $G_{\Lambda}\left(q^{2}\right)$ in Eq. 76 to express the deviation of mon-proton elastic scattering from electron-proton elastic scattering. In doing so we are making an assumption to which we shall return at the end of the article. We are assuming that the devlation between mon-proton and electron-proton elastic scattering will increase as $\left|q^{2}\right|$ increases.

Nonrelativistic quantum mechanles provides some insight into the relation between the anomalous interaction concept and the form factor concept. 89 
Nonrelativistically the form factor of Eq. 770

is the three-dimensional

Fourier transform of

$$
\rho_{\mu}(r)=\frac{1}{4 \pi r} e^{-r \Lambda}
$$

Thus the discovery of a form factor for the muon of the type of Eq. $77 \mathrm{~b}$ could also be interpreted as the discovery of an anomalous interaction of the Yukawa form with range $1 / \Lambda_{\mu}$.

In all of this we have assumed that the electron is a pure Dirac point particle. There is no need for this assumption. We can ascribe a form factor $G_{e}\left(q^{2}\right)=1 /\left(1+\left|q^{2}\right| / \Lambda_{e}^{2}\right)$ to the electron. Then to order $\left|q^{2}\right|$

$$
\frac{G_{\mu}\left(q^{2}\right)}{G_{e}\left(q^{2}\right)}=\frac{1+\left|q^{2}\right| / \Lambda_{e}^{2}}{1+\left|q^{2}\right| / \Lambda_{\mu}^{2}} \approx \frac{1}{1+\left|q^{2}\right| / \Lambda_{d}^{2}}
$$

where

$$
\frac{1}{\Lambda_{d}^{2}}=\frac{1}{\Lambda_{\mu}^{2}}-\frac{1}{\Lambda_{e}^{2}}
$$

Then $\Lambda_{\mathrm{d}}$ simply measures a difference in behavior between the electron and the muon. From now on we shall use $\Lambda_{\mathrm{d}}$. Defining

$$
\rho_{\text {elastic }}\left(q^{2}\right)=G_{\mu}^{2}\left(q^{2}\right) / G_{e}^{2}\left(q^{2}\right)=l / 1+\left|q^{2}\right| / \Lambda_{d}^{2}
$$

Eq. 76 becomes

$$
P_{\text {elastic }}\left(q^{2}\right)=\left(d \sigma / q^{2}\right)_{\mu p, e l a s} /\left(d \sigma / d q^{2}\right)_{e p, e l u s}
$$

Eq. $80 \mathrm{~b}$ is not exactly true, there is a slight correction due to the muon mass which is not explictly exhibited.
In the experimental determination of $\rho_{\text {elastic' }}$ the absolute normalizations of the two cross sections present a special problem because of the very different lechniques used to measure the cross sections. The electron cross section measurements use 87 a high intensity beam, short hydrogen target, and small angle spectrometer. The muon experiments use $\mathrm{9}^{91-94}$ a low intensity beam, long hydrogen target and large solid angle detector. To allow for relative normalization uncertainties, Eq. $30 \mathrm{a}$ is changed to the form

$$
\rho_{\text {elastic }}\left(q^{2}\right)=\frac{N^{2}}{\left[1+\left|q^{2}\right| / \Lambda_{d}^{2}\right]^{2}}
$$

Table I presents the fits to Eq. (80c) of the three comparisons $91-93$

\begin{tabular}{|c|c|c|c|c|}
\hline \multirow{2}{*}{$\frac{\left\langle\begin{array}{c}\mathrm{p}_{\text {incident }} \\
(\mathrm{GeV} / \mathrm{c})\end{array}\right.}{5.8}$} & \multicolumn{2}{|l|}{$\begin{array}{c}q^{2} \\
(\mathrm{GeV} / \mathrm{c})^{2}\end{array}$} & $\mathbf{N}$ & $\begin{array}{c}1 / \Lambda^{2} \\
(\mathrm{GeV} / \mathrm{c})^{-2}\end{array}$ \\
\hline & Ref. 93 & 1.02 & $1.07 \pm 0.09$ & $+0.042 \pm 0.046$ \\
\hline $7 \cdot 3$ & Ref. 93 & 1.52 & $0.97 \pm 0.10$ & $+0.034 \pm 0.042$ \\
\hline 5.8 & $\begin{array}{l}\text { Ref. } 93 \\
\text { recoil protons }\end{array}$ & 0.95 & $1.00 \pm 0.10$ & $+0.037 \pm 0.066$ \\
\hline 2.1 & Ref. 91 & 0.62 & $0.97 \pm 0.08$ & $+0.107 \pm 0.075$ \\
\hline $6,11,17$ & Ref. 92 & 0.26 & $0.96 \pm 0.03$ & $+0.054 \pm 0.051$ \\
\hline
\end{tabular}
which has been done

TABLE I

Least-squares fit parameters to world data of $\mu-p$ elaslic scattering. The normalization has been constrained to $N=1.0 \pm 0.1$. Taken from Ref. 93.

Mon-electror. universality requires

$$
\rho_{\text {elastic }}\left(q^{2}\right)=1 \text {, for all } q^{2}
$$


Hence it requires

$$
1 / \Lambda^{2}=0
$$

regardless of normalization problems.

Table I shows that no individual experimental determination yields a statistically significant deviation from Eq. 82. However the combined data 93 from these experiments, Eq. 83, shows some weak cvidence for a deviation.

$$
1 / \Lambda^{2}=+0.051 \pm 0.024(\mathrm{GeV} / \mathrm{c})^{-2}
$$

This is not a strong effect - only two standard deviations - certainly not strong enough to seriously challenge muon-electron universality. Still, we should not ignore Eq. 83. We return to it in Sec. XII.
X. COMPARISON OF ELECTRON-PROTON AND MUON-PROTON INEIASTIC SCATTERING

\section{A. Theoretical Background}

Another very general way to search for anomalous behavior of the electron or the muon is to compare electron-proton and muon-proton inelistic scattering. The kinematics of lepton-proton inelastic scattering are a bit complicated and we shall digress for a moment to discuss the experimental method and kinematics. The relevant kinematics, for the mon case, are shown in Fig. 23 for one-photonexchange.

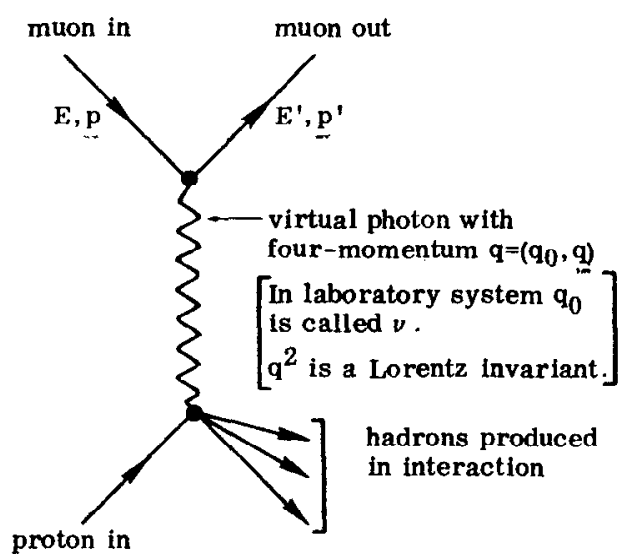

$M$ is proton mass

F1g. 23

In the experiments used in the comparison only the inelastically scattered charged lepton is detected. No attempt is made to detect any of the hadrons produced. This inelastic scattering experiment then sums experimentally over the different hadronic states which can be produced. As may be deduced from Fig. 23 the reaction is then completely described by three independent kinematic quantities. For using $p, p^{\prime}$, and $P$ to represent the four-momentum of the incident lepton, final lepton and incident proton respectively, we can define the 
four-momentum of the virtual photon

$$
q=p-p^{\prime}
$$

and the three independent Lorentz scalars

$$
s=(p+P)^{2}, \quad q^{2}=\left(p-p^{\prime}\right)^{2}, P \cdot q=p \cdot\left(p-p^{\prime}\right)
$$

However it turns out to be more comenient to use another set of independent quantities

$$
E, q^{2}, v=E-E
$$

$E$ is the laboratory energy of the incident lepton and $v$, the laboratory energy of the virtual photon, represents a Iorentz scalar since $v=p \cdot q / M$. The experiment consists of the measurement of the double differential cross section of the inelastically scattered lepton. This differential cross section, $d^{2} \sigma / d q^{2} d v$, is a function of $E, v$ and $q^{2}$.

B. Experimental Results

To compare muon-proton $(\mu p)$ inelastic scattering with electron-proton (ep) inelastic scattering we again define the ratio

$$
\left.\rho_{\text {inelastic,p }}\left(\mathrm{q}^{2}, v\right)=\left[\mathrm{d}^{2} \sigma / \mathrm{dq}^{2} \mathrm{~d} v\right)_{\mu \mathrm{p}} /\left(\mathrm{d}^{2} \sigma / \mathrm{dq} \mathrm{q}^{\sigma} \mathrm{d} v\right) \mathrm{ep}\right]
$$

The effect of the muon-electron mass difference, which must be taken into account, is not shown explicitly.

Two medium energy muon-proton inelastic scattering experiments, one at SIAC by Hraunstein et a $1.95,96$ and one at BNL by tintenberg et a1. 94,97 nave been carried out. No statistically significant deviations from $p_{\text {inelastic }}\left(q^{2}, v\right)=1$ have been found in any portion of the $q^{2}-v$ kinematic region. Furthermore no statistically significant deviation have been found if the data is summed over one of the variables, $q^{2}$ or $v$, a search being made for a deviation from $p_{\text {inelastic }}=1$ in the other variable.

The same experimenters who measured mon-proton inelastic scattering at $\mathrm{BNL},{ }^{97}$ have also measured mon-deuteron inelastic scattering and compared it ${ }^{96}$ with electron-deuteron inelastic scattering. They find no statistically significant dependence of $\rho_{\text {inelastic, } d}$ on $q^{2}$ or on $v$; but they do find that the average value of $\rho_{\text {inelastic, } d}$ is less than 1 by about 2 standard deviations.

Finally, the recent very high energy ( 53 and $150 \mathrm{GeV}$ ) muon-proton inelastic scattering experiment of Fox et al. ${ }^{99}$ at FNAL can be used to test muon-electron universality if the inelastic structure functions $v W_{2}$ and $W_{1}$ are tunctions only of $W=2 M V /\left|q^{2}\right|$ - that is if one assumes Bjorken scaling. ${ }^{89}$ Scaling must be assumed because there is no electron-proton inelastic scattering data at that energy. This experiment is a potentially important test of mon-electron universality because $\left|q^{2}\right|$ values up to $50(\mathrm{GeV} / \mathrm{c})^{2}$ are obtained, whereas the experiments discussed above only reach $\left|q^{2}\right|$ values of 3 or $4(\mathrm{GeV} / \mathrm{c})^{2}$. However uncertainties about scaling at the 10 or $20 \%$ level prevent precise use of this data; and once again one finds no statistically significant violation of monelectron universality.

To quantify the observations we have just made and to connect the inelastic scattering comparison with the elastic comparison, we define

$$
p_{\text {inelastic }}\left(q^{2}, v\right)=N^{2} /\left[I+\left|q^{2}\right| / \Lambda^{\prime}\right]^{2}
$$

just as in Eq. $(80 c)$. But here we average over $v$ to obtain $N^{\prime}$ and $\Lambda^{\prime}$. The parameters are primed to indicate that they apply to inelastic scattering. Again $\Lambda^{\prime-2}=\Lambda_{e}^{\prime-2}-\Lambda_{e}^{\prime-2}$. Table II gives the values of $N^{\prime}$ and $\Lambda_{d}^{\prime}$ found in the inelastic experiments. 
TABLE II. Fits to Eq. 85b for comparing muon-hadron and electron-hadron inelastic scattering. The $150 \mathrm{GeV} / \mathrm{c}$ data assumes Bjorken scaling of the inelastic structure functions.

\begin{tabular}{cccccc}
\hline $\begin{array}{c}\left\langle\mathrm{p}_{\text {incident }}\right\rangle \\
\text { GeV/c }\end{array}$ & target & Ref. & $\begin{array}{l}\left|\mathrm{q}^{2}\right| \text { range } \\
(\mathrm{GeV} / \mathrm{c})^{2}\end{array}$ & $\mathrm{~N}^{\prime}$ & $\begin{array}{c}1 / \Lambda^{2} \\
(\mathrm{GeV} / \mathrm{c})^{-2}\end{array}$ \\
\hline 5.8 and 7.3 & $\mathrm{p}$ & 97 & $0.4-3.6$ & $0.997 \pm 0.043$ & $+0.006 \pm 0.016$ \\
12 & $\mathrm{p}$ & 96 & & $0.946 \pm 0.042$ & $+0.021 \pm 0.021$ \\
150 & $\mathrm{p}$ & 99 & $6-44$ & $1.10 \pm 0.12$ & $+0.004 \pm 0.003$ \\
7.3 & $\mathrm{~d}$ & 98 & $0.4-3.4$ & $0.925 \pm 0.038$ & $-0.019 \pm 0.016$ \\
\hline \hline
\end{tabular}

Hence we find no statistically significant deviation from muon-electron universality in charged lepton-hadron inelastic scattering.
XI. CHARGED LEETTON FORM FACTORS IN COLLIDING BEAM EXPERIMENTS

A. Elastic Electron-Electron Scattering: $e^{-}+e^{-} \rightarrow e^{-}+e^{-}$

This reaction occurs through the diagram in Fig. 24

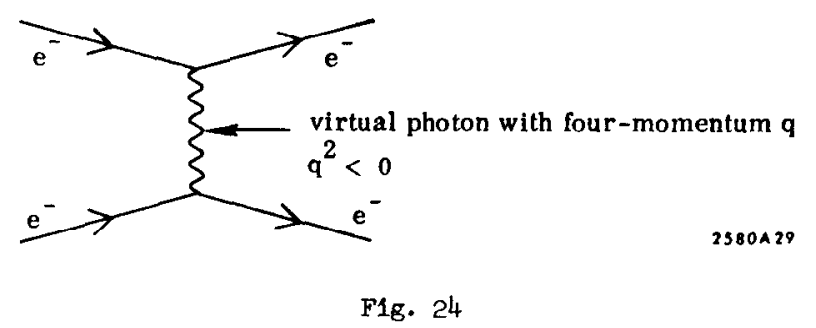

To obtain larger values of $\left|q^{2}\right|$ it is necessary to use an electron-electron colliding beam apparatus, and such an experiment has been carried out by Barber et al. ${ }^{100}$

If we ascribe an electron form factor

$$
g_{e s}\left(q^{2}\right)=1 /\left[1-q^{2} / \lambda_{e s}^{2}\right],
$$

as in Eq. $77 \mathrm{~b}$, the differential cross section predicted by pure quantum electrodynamics will be nultiplied by the factor $I /\left[1-q^{2} / \lambda_{e s}^{2}\right]^{4}$. The $\lambda_{\text {es }}$ parameter in this form factor is lower case to distinguish it from the $\Lambda_{e}$ parameter which occurs in Eq. 79 . The latter applies to electron-proton elastic scattering. An anomalous electron-proton interaction could lead to an electron form factor differeing from unity in electron-proton elastic scattering, but would not effect electron-electron scattering. Therefore $\lambda_{\text {es }}$ and $\Lambda_{e}$ need not be the same. The $s$ subscript indicates that $q^{2}$ is negative and hence spacelike in the diagram in Fig. 24. As we see next, an electron-virtual photon vertex with $q^{2}$ positive and hence timelike can also occur; and we allow a different form factor in that case. 
Barber et al. ${ }^{100}$ find with $95 \%$ confidence the lower limit

$$
\lambda_{\text {es }}>6.1 \mathrm{GeV} / \mathrm{c}
$$

Thus this experiment agrees with the assumption that the electron is a point particle.

B. Bhabha Scattering: $e^{-}+e^{+} \rightarrow e^{-}+e^{+}$

This process takes place through the two diagrams in Fig. 25.

(o)

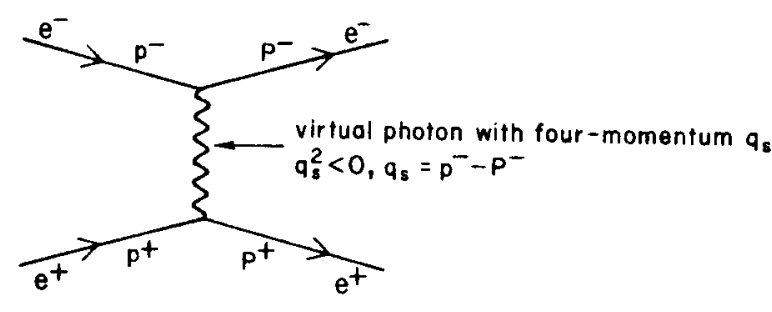

(b)

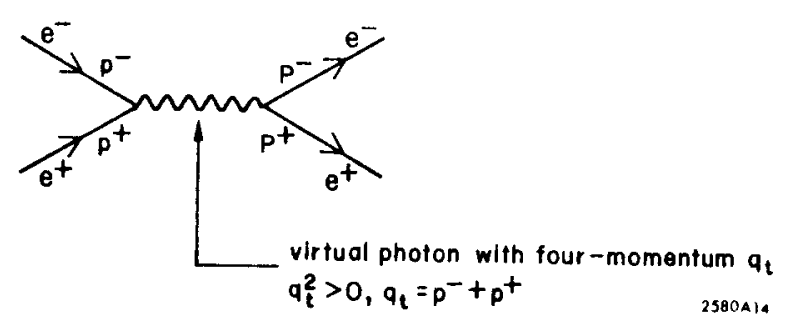

Fig. 25

We could ascribe the space-like form factor $g_{\text {es }}$, Eq. $86 a$, to each vertex in the diagram in Fig. $25 \mathrm{a}$; and the time-like form factor

$$
g_{\text {et }}\left(q^{2}\right)=1 /\left[1-q^{2} / \lambda^{2} \text { et }\right]
$$

to each vertex in Fig. 25k. But Eq. $86 \mathrm{~b}$ is a bit peculiar because now $q^{2}>0$; ience, we seem to be looking for an enhancement in the cross section, rather than the reduction due to a conventional form factor. It seems best to allow either possibility by defining

$$
\begin{aligned}
& \mathrm{g}_{\text {es }}^{ \pm}=I /\left[1+q^{2} / \lambda_{\text {es }}^{ \pm 2}\right] \\
& \mathrm{g}_{\text {et }}^{ \pm}=I /\left[1+q^{2} / \lambda_{\text {et }}^{ \pm 2}\right]
\end{aligned}
$$

As we shall see in a moment, we find $\lambda \gg\left|q^{2}\right|$ in all experiments. Thus we are really making a linear fit

$$
g \approx 1+c q^{2}
$$

witi $c$ allowed to be positive or negative.

Many electron-positron colliding beam experiments on Bhabha scattering have been carried out. ${ }^{101-103}$ They all agree wilh the conventional view of the electron as a point particle in both the space-like and time-like regions. We give in Table III the lower limits on the $\lambda^{\prime}$ 's obtained in recent experiments at SIAC. ${ }^{102,10}$ These being the highest energy and largest statistics experiments yet performed, are the most sensitive.

\section{TABLE III}

Lower limits with $95 \%$ confidence on $\lambda_{\text {es }}^{ \pm}$and $\lambda_{\text {et }}^{ \pm}$in Eqs. (86c) and (86d). $\lambda$ in unites of $\mathrm{GeV} / \mathrm{c}$

\begin{tabular}{llllllll}
\hline & \multicolumn{2}{l}{ Lower limit on } & & & & & \\
$\lambda_{\text {es }}^{+}$ & $\lambda_{\text {es }}^{-}$ & $\lambda_{\text {et }}^{+}$ & $\lambda_{\text {es }}^{-}$ & if $\lambda_{\text {es }}^{+}=\lambda_{\text {et }}^{+}$ & if $\lambda_{\text {es }}^{-}=\lambda_{\text {et }}^{-}$ & Ref. \\
\hline 14 & 14 & 10 & 17 & 16 & 13 & 103 \\
& & & 23 & 14 & 102 \\
\hline
\end{tabular}




\section{Muon Pair Production: $\mathrm{e}^{-}+\mathrm{e}^{+} \rightarrow \mu^{-}+\mu^{+}$.}

This process takes place only through the diagram in Fig. 26 and thus involves only time-like form factors. Several colliding beam studies of this reaction have been made. ${ }^{102-104}$ They all agree with the assumption that the mion is pointlike in the time-like region.

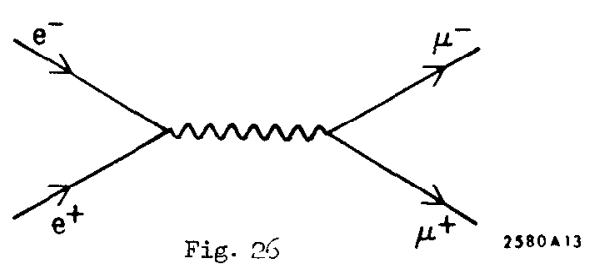

Using Eq. 86d, only $g_{\mu t}^{ \pm}$occurs in Fig. 26 , and allowing different $\lambda$ 's for the muon and electron; experiments 102,103 show

$$
\lambda_{\mu \mathrm{s}}^{ \pm} \geq 15 \mathrm{GeV} / \mathrm{c}
$$

with $9 \%$ confidence. Thus the muon also acts like a point particle in the reaction $\mathrm{e}^{+}+\mathrm{e}^{-} \rightarrow \mu^{+}+\mu^{-}$; and muon-electron universality is maintained in the lepton pair production reaction $e^{+}+e^{-} \rightarrow l^{+}+l^{-}$.

\section{SPECULATIONS}

We have come to the end of this lons review of experiments without coming upon any new facts or even new clues as to the nature of the muon and electron or as to their relation to each other. Table I in the Introduction still encompasses all that is known of the charged leptons. Nevertheless physics is an experimental science and experiments seeking new facts or new clues about heavy leptons will go on. Therefore in our view it seems useful to speculate as to what might be the most fruitful experimental directions or at least to give our prejudices as to fruitful directions.

\section{A. Searches for Heavy Leptons}

Those who have searched for heavy leptons, the authors included, have to some extent ignored the probable dominance of the $v+$ hadrons decay modes. We have concentrated on the leptonic and single pion decay modes because we understood how to search for these modes. But as the searches extend to higher lepton masses, the search sensitivities may decrease drastically unless we learn how to detect the multi-hadron decay modes. We need some new experimental ideas in this direction.

\section{R. Charged-Lepton Form Factors}

In the original version of this paper written about three years ago we speculated that the muon electromagnetic form factor might deviate from 1 . This speculation was based (1) on the earlier mon-proton elastic and inelastic scattering measurements, Refs. 91,92 and 95 , all having $N$ or $N^{*}$ less than 1 ; and (2) was allowed by the precision of then existing measurements of $\lambda_{\text {es }}^{ \pm}$, $\lambda_{\text {et }}^{ \pm}$, and $\lambda_{\mu \mathrm{s}}^{ \pm}$in $\mathrm{e}^{+} \mathrm{e}^{-}$colliding beam experiments. However the recent measurements of these quantities, Sec. XI, yield $95 \%$ confidence lower limits of about $15 \mathrm{GeV} / \mathrm{c}$. This is to be compared with the possible values of $\Lambda$ or $\Lambda^{\prime}$ in the elastic or inelastic scattering comparisons (Sec. $\mathrm{XX}$ and $\mathrm{X}$ ) which are of the order of $5 \mathrm{GeV} / \mathrm{c}$. 
Therefore it is unreasonable to attempt to attribute any possible anomalies in the elastic or inelastic scattering comparis ons to a form factor effect at the virtual photon-lepton vertex.

The new $e^{+} e^{-}$colliding beam measurements of $\lambda_{\text {es }}^{ \pm}, \lambda_{\text {et }}^{ \pm}$and $\lambda_{\mu s}^{ \pm}$also surpass in precision the limit on the muon form factor set by the measurement of the muon's gyromagnetic ratio, $g_{\mu}$ (Sec. VI. B). This limit is set through the diagram in Fig. 27

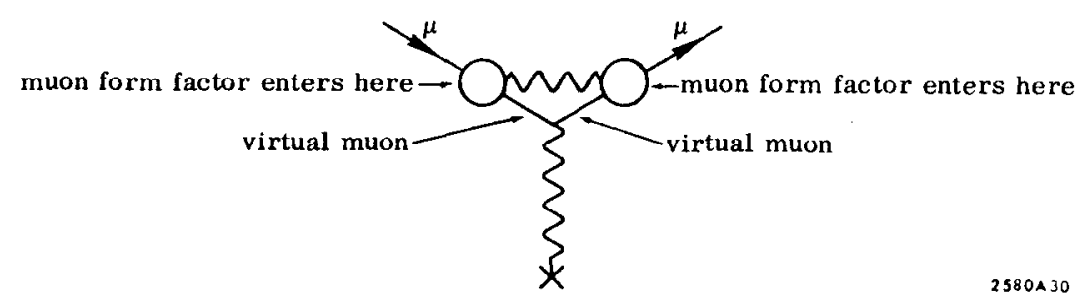

Fig. 27

The $q^{2}$ which enters the muon form factor in this diagram comes from the virtual muons, and the important $q^{2}$ values are those whose magnitude is smaller than $\mathrm{m}_{\mu}^{2} ; \mathrm{m}_{\mu}$ is the muon mass. Hence the measurement of $\mathrm{g}_{\mu}$ both space-like and time-like regions must be selected. It is conventional to select the form factor given by Eq. 86a. The $\mathrm{g}_{\mu}$ measurement requires ${ }^{1,105}$ that in Eq. 86a.

$$
\lambda_{\mu \mathrm{g}}>7.0 \mathrm{GeV} / \mathrm{c}
$$

with $95 \%$ confidence. The $\lambda_{\mu g}$ lower limit in Eq. 89 is less than half the $\lambda_{\mu \mathrm{s}}^{+}$ given in Eq. 83.

Of course from a very general viewpoint we should not compare a very high precision, very small $\left|q^{2}\right|$ search for a non-unity form factor with a relatively lower precision, very high $\left|q^{2}\right|$ search. A deviation in the form factor from unity could occur in one $q^{2}$ range and not in the other $q^{2}$ range. And the formula $\mathrm{N} /\left(1 \pm\left|\mathrm{q}^{2}\right| / \lambda^{2}\right)$ used to test for deviations has no physical significance until a deviation is found. Therefore it seems best to regard E'qs. 88 and 89 as complementary measurements.

To summarize: all electrodynamic measurements confirm unity form factors for the electron and muon. There is not even a hint of a deviation from the simple concept that the electron and muon and point Dirac particles. Nor is there even a hint of deviation from muon-electron universality in electromagnetic reaction.

\section{Anomalous Lepton-Hadron Interactions}

Indeed the reactions which show even a hint of a deviation from muon-electron universality are those which take place in the presence of a hadron. There are two such reactions which have been studied:

1. In charged lepton-proton elastic scattering, Sec. $\perp X$, there is a two standard deviation effect -- the muon-proton differential cross section decreases more rapidly than the comparable electron-proton cross section as $\left|q^{2}\right|$ increases.

2. In the charged lepton-hadron inelastic scattering comparison, Sec. $X$, $N^{\prime}$ averages less than 1 for the three medium energy experiments. The use of the $150 \mathrm{GeV}$ experiment to test muon-electron universality is provisional until possible deviations from scaling are better understood.

Thus we are left with the vague possibility that there is an anomalous electron-hadron or an anomalous muon-hadron interaction or both -- these anomalous interactions resulting in mon-hadron cross sections being slightly less than comparable electron-hadron cross sections. By anomalous we mean that the interaction is neither that described by standard quantum electrodynamics, nor is it a weak interaction. 


$$
\text { . } \cdots
$$

As a speculative example for this vague possibility suppose that the muon has a special interaction with the hadrons, an interaction not possessed by the electron. Muon-proton elastic or inelastic scattering would take place through the sum of the two diagrams in Fig. 28

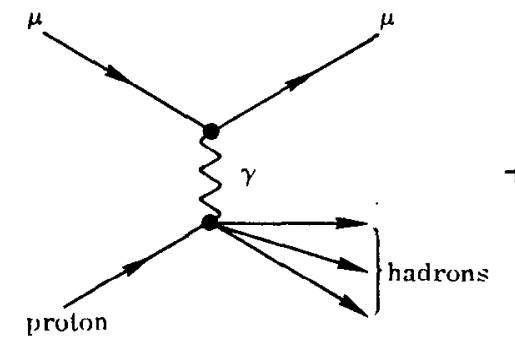

One-photon exchange

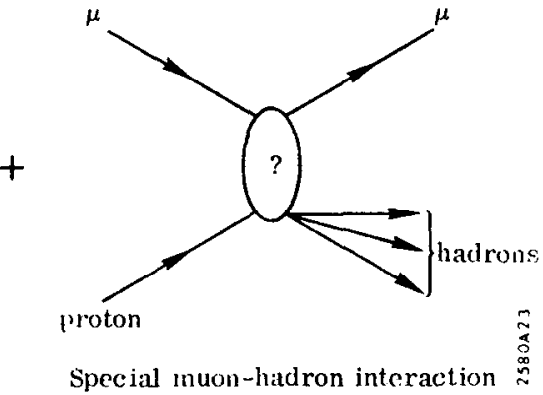

Fig. $;\}$
The second diagram would result in a difference between muon-proton and electronproton inealstic cross sections, because only the first diagram would enter in electron-proton inc? astic scattering.

The problem is how to find this special interaction -- how to enhance the effects of this special interaction. The usual prescriptions are to measure to greater precision or to carry out reactions at greater $\left|q^{2}\right|$ values. But at present we don't know how to improve the precision of the elastic and inelastic comparisons. Systematic errors at the $5 \%$ level plague the experiments.

And there is no guarantee that larger $\left|q^{2}\right|$ values will enhance the anomalous effect. For example, assume that the muon inter xcts with the hadrons through the exchange of particle $X$ with spin $I$ and mass $M_{x}$ as in Fig. 29.

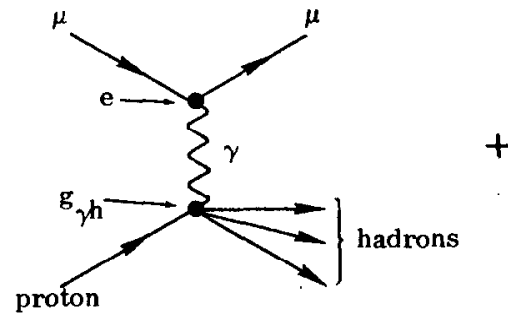

One-photon exchange

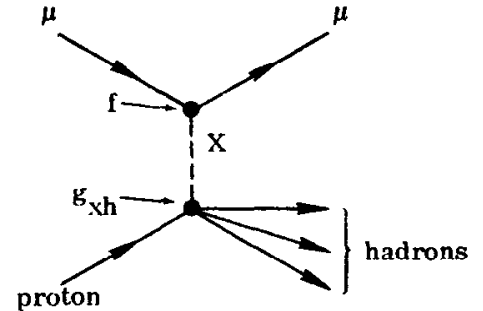

one-X exchange

$2580 \wedge 24$
Fig. 29

A conventional speculation 106,107 is that the $X$ particle is some undiscovered heavy photon, but we prefer the speculation that the $X$ particle is itself a hadron. More generally the $X$ particle might be taken to represent the summation of the interaction of different kinds of hadrons with the moon. The coupling constants are indicated in the diagrams; thus $e$ is the electric charge. Those at the lower vertices are to be regarded only as very crude measures of the strength of the coupling of the virtual photon or the $X$ particle to hadrons. Then 106

$$
\rho_{\text {elastic }} \text { or } \rho_{\text {inelastic }} \approx\left[I+a\left(\frac{\left|q^{2}\right|}{\left|q^{2}\right|+M_{x}^{2}}\right)\right]
$$

$$
a=\left(\frac{f}{e}\right)\left(\frac{B_{x n}}{B_{\gamma n}}\right)
$$

Suppose that $b \sim-0.05$ and $M_{x} \sim 5 \mathrm{CreV} / \mathrm{c}^{2}$. Then we can get the deviations possibly seen in the elastic and inelastic comparisons; yet even for $\left|q^{2}\right| \gg M_{x}^{2}$ we only get a $10 \%$ effect. 
Nor would this special interaction be easy to find in other reactions. To estimate the experimental limits of $f$, the coupling of the muon to the hadron $x$, take $\left(g_{x h} / g_{y h}\right)^{2}$ to be the ratio of a typical hadron hadron cross section (30 mb) to the photon-proton total cross section $(0.12 \mathrm{mb})$. As discussed above, exisiting muon-proton scattering measurements easily allow b to be as large as .05 . Then

$$
|f / e| \lesssim 0.0 \% / \sqrt{250} \lesssim 1 / 300
$$

Thus in this "X=hadron" model, the coupling of the muon to the hadrons is much weaker than the electromagnetic coupling. The contribution of this special. interaction to e lectromagnetic processes such as $\mathrm{e}^{+}+\mathrm{e}^{-} \rightarrow \mu^{+}+\mu^{-}$would be $10^{-7}$ of the one-photon exchange process, Fig. 26.

Returning to the general example of Fig. 28, or to the converse example of a special electron-hadron interaction, how then is one to isolate this interaction? What is needed is a pair of reactions in which the one-photon exchange process is suppressed. Perhaps such a reaction pair is

$$
\begin{aligned}
& p+p \rightarrow \mu^{+}+\mu^{-}+\text {hadrons } \\
& p+p \rightarrow e^{+}+e^{-}+\text {hadrons }
\end{aligned}
$$

the reactions discussed in Sec. V.D as a background source. Very preliminary data $^{62-64}$ indicates that the muon-electron ratio is roughly one. Still for the immediate future the reactions in Eq. 91 are perhaps the best comparative experimental way to search for anomalous interactions of charged leptons with hadrons.

We emphasized the phase comparative experimental in the last sentence because there has been a good deal of speculation ${ }^{108}$ recently as to whether the large $c^{+}+\mathrm{c}^{-} \rightarrow$ hudrons cross section -- large compared to simple parton model predictions (Secs. III.A and V.B) -- indicated an anomalous electron-hadron interaction. This speculation is that in addition to the one-photon exchange diagram of Fig. 30a there is a special electron-hadron interaction which leads to the diagram in Fìg. 30 .

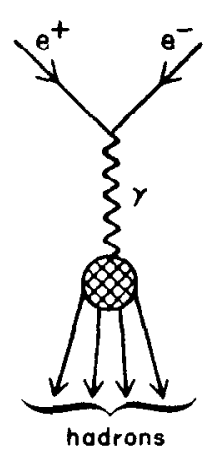

(a)

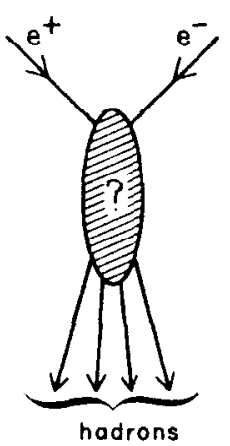

(b)
Fig. 30

However this is not a comparalive experimental search for a special electron-hadron interaction because we have no sure theory for calculating the cross hatched photon-hadron vertex in Fig. 30\%. It is only the use of a simple parton model for calculating that vertex which leads to the invoking of the diagram in Fig. $30 \mathrm{~b}$ It may be that the high energy reactions

$$
e^{+}+e^{-} \rightarrow \text { hadrons }
$$

will turn out to be the best way to study anomalous charged lepton-hadron interactions; but first we have to corrince ourselves that we cannot explain the cross sections for these reactions using corventional theory. 
REFERENCES

1. F. Ficasso in High-Energy Physics and Nuclear Structure, 1970 (Plenum Press, New York, 1970), this article also reviews other tes ls of muonelectron universality; J. Beiley et al., Nuovo Cimento, 9A, 369(1972) .T. Bailey et al., Phys. Letters 28B, $287(1.968)$.

2. S.T. Brodsky in Proceedings of the 4th International Symposium on Electron and Photon Interactions at High Fnergy, Daresbury, England (1969); S.1. Brodsky and S.D. Drell, Ann. Rev. Nucl. Sci. 20, 147(1970). These articles review quantum electrodynamic tests of muon-electron universality.

3. T.T. Lee and C.S. Wu, Ann. Rev. Nucl. Sci. 15, 381(1965). This article reviews weak interaction tests of muon-electron universality.

4. G. Feinberg and L.M. Lederman, Ann. Rev. Nucl. Sci. 13, 431(1963).

5. F.F. Low, Phys. Rev. Letters 14, 238(1965).

6. 1.0. Rarut et al., Phys. Rev. 182, 1844(1969).

7. The notation used here is that given in J.D. Bjorken and S. Drell, Relativistic Quantum Mechanics (McGraw-Hi11, New York, 1964) or in M.L. Perl, High Fnergy Hidron Physics (Wiley, New York, 1974).

@. E. Iipmanov, 7h. Eksp. Teor. Fiz. 43, 893(1962) (Sov. Phys. JETP 16, 634 (1963)).

a. Ya. B. Zel'Dovich, Usp. Fiz. Nauk. 78, 549(1962) (Sov. Phys. Usp. 2, 931(1963)).

10. S.S. Gerstein and V.N. Folomeshkin, Yad. Fiz. $\underline{8}$, 768(1968) (Sov. J. Nucl. Phys. \&, $447(1969))$.

11. B. Pontecorve, th. Eksp. Teor. Fiz. 23, 1717(1967) (Sov. Phys. JETP 26, $984(1968))$.

12. K.:. Marshak, Rizzuddin, and C.P. Ryan, Theory of Weak Interactions in Purticle Fhysics (Interscience, New York, 1069).
13. E. T. Konopinski and H.M. Mahmoud, Phys. Rev. 22, 1045(1953).

14. L.S. Kisslinger, Phys. Rev. Letters 26, 998(1971).

15. D. A. Bryman et a‥, Phys. Rev. Letters 28, 1469(1972).

16. It would be pointless to attempt to list here even the most important papers in the vast literature on these theories. Recent review papers with extensive lists of references are:

E.S. Abers and B.W. Lee, Phys. Reports 9C, 1(1973)

M.A.B. Beg and S. Sirlin, Gauge Theories of Weak Interactions, Rockefeller Univ. Report No. COO-2232B-47 (1974), to be published in the Annual Review of Nuclear Science, Vol. 24.

17. Two papers of particular value in summarizing the position of heavy leptons in these theories are the J.D. Bjorken and C.H. Llewellyn Smith, Phys. Rev. D7, 88(9773) and C.H. Llewellym Smith in Proceedings of the Fifth Hawaii Topical Conference in Particle Physics (1973) Vol. 1 (Univ. of Hawail Press, Honolulu, 1974).

18. C.H. Albright, C. Jarlskg, M. O. TJia, CERN preprint TH-1887 (1974), as well as the first paper of Ref. 17 provide an extensive list of theoretical models and their associated heavy 7 pptons. We chose to discuss only the more conventional models that include only the pairs mentioned in the text.

19. J.R. Primack and H.R. Quinn, Fhys. Rev. D6, 3171(1972).

20. S.s. Gershtein et al., Institute of High Energy Physics Report No. IHEP 72-115.

21. A. Barna et al.. Phys. Rev. 173, 1391(1968); M. L. Perl, Proceedings of the 1967 International Symposium on Electron and Photon Interactions at Jigh Energies (Stanford Linear Accelerator Center, Stanford, 1967).

22. See for example: B. Pontecorvo, Th. ETT. Pis. Red. 13, 218(1971) (Sov. Phys. JEIP Letters 13, 199(1971)).

23. Y.S. Tsai, Phys. Rev. D4, 2821(1971).

\section{$-81-$}


24. E.M. Lipmanov, Zh. Eksp. Teor. Fiz. 46, 1917(1964) (Sov. Phys. JETP 14, $859(1962))$.

25. E.W. Beier, Lettere Nuovo Cimento 1, 1118 (1971).

26. A.K. Mann, Lettere Nuovo Cimento 1 , 486(1971).

27. J.J. Sakurai, Lettere Nuovo Cimento 1, 624(1971).

28. H. B. Thacker and J.J. Sakurai, Phys. Letters 36B, 103 (1971).

29. K.W. Rothe and A.M. Wolsky, Nucl. Phys. 10B, 241(1969).

30. G. Tarnopolsky et al., Phys. Rev. Letters 32, 432(1974).

31. B. Richter Proceedings of the 17th International Conference on High Energy Physics, 1974 (to be published).

32. C.A. Lichtenstein, Thesis, Harvard University (1970), unpublished.

33. E.W. Beier, Thesis, University of Illinois (1966), unpublished.

34. C.A. Ramm, Nature 227, 1323(1970); Nature Physical Science 230, 145(1971); Addendums 2 through 6 to CERN Report No. NPA 69-6 (1971-1972).

35. A.D. Liberman et al., Phys. Rev. Letters 22, 663(1969).

36. A.D. Liberman, Thesis, Harrard University (1969), unpublished. In this thesis Liberman notes the evidence for the $\mu^{ \pm}$in $\mu^{ \pm} \gamma$ mass spectrum, but comments that its statistical significance is only 2 standerd deviations.

37. A. R. Clark, Nature 237, 383(1972).

38. D.H. Coward et al., Phys. Rev. 131 1782(1963).

39. This search was only definitive up to a mass of about $.4 \mathrm{GeV} / \mathrm{c}^{2}$. For higher masses the lifetime of the heavy sequential lepton would be too short.

40. R. E. Ansorge et al., Phys. Rev. 17, 26(1973).

41. D. E. Dorfan et al., Phys. Rev. Letters 14, 999(1965), P. Franzini et al., Phys. Rev. Letters 14, $196(1965)$.
42. B. Alper et al., Experiments on High Energy Particle Collisions, (American Institute of Physics, New York, 1973).

43. J.W. Cronin ct al., Enrico Fermi Institute Preprint EFI-74-2.9 (1974).

44. S. Franke] et al., Phys. Rev. D9, 1932(1974).

45. V. Alles-Borelli et al., Lettre Nuovo Cimento 4, 1156(1970); M. Bernardini et al. , Nuovo Cimento I7, 383(1973).

46. J. Feller et al., paper presented to the $17 \mathrm{th}$ International Conference on High Energy Physics, London, 1974).

47. C. Bacci et al., Phys. Ietters 44B, 530(1973).

48. G. Hanson et al., Lettere Nuovo Cimento I, 587(1973).

1;9. M. L. Perl, Bull. Amer. Phys. Soc. 19, 542(1973).

50. The luminosity of a colliding beam facility is the quantity which when multiplied by a reaction cross section gives the number of events produced by that reaction per unit time. The maximu depends on the beam energy -- we are using a corvenient average value here.

51. J. Kirkby et al., SLAC Proposal SP-18 (1974), unpublished.

5?. See for example the papers presented by A. Ali and by A. Soni to the 17 th International Conference on High Fnergy Physics -- London, 1974.

53. K.J. Kim and Y.S. Tsai, Phys. Letters 40B, 665 (1972).

54. Some classic references to photoproduction of particle pairs are W. Pauli and V.F. Weisskopf, Helv. Phys. Acta I, 709(1934); and W. Heitler, Quantum Theory of Radiation (Clarendon Press, Oxford, England, 1954).

55. A. Rothenberg, Thesis, Stanford University (1962), unpublished.

56. C.A. Heusch and J. Sandweiss, 1969 Summer Study for Nati onal Accelerator Laboratory, Vol. 4, edited by A. Roberts (national Accelerator Laboratory, Batavia, Illinois, 1970), p. 111.

57. M. Schwartz, Reports on Progress in Physics 27, 61(1965).

58. W. Lee et a1., Proposal No. 87A to the National Accelerator Laboratory (1970), unpublished. 
59. S.D. Drell and T.M. Yan, Phys. Rev. Letters 25, 316(1970) and references contained therein; Ann. Phys. N.Y. 66, 578(1971).

60. S.M. Berman et al., Phys. Rev. D4, 3388(1971).

61. G. Ghiu and J.F. Gunion, LBLL Report No. IBI-3020 (1974), submitted to Phys. Rev.

62. J.A. Appel et al., Phys. Rev. Letters 33, 722(1974).

63. J.P. Boymond et al., Phys. Rev. Letters $33,112(1974)$.

64. F.W. Busser et al. , Proceedings of the 17th International Conference on High Energy Physics, 1974 (to be published).

65. J.H. Christenson et al., Phys. Rev. Letters 25, 1523(1970).

66. For a specific calculation of this process and an analysis of the search results, scc F. Gutbrod and D. Sch1ldsnecht, zeit. Phys. 142, 271(1966).

67. C. Betourne et al., Phys. Letters 17. 70(1965).

68 . H. J. Behrend, Phys. Rev. Letters 15, 900(1965).

69. R. Budnitz et a1., Phys. Rev. 141, 1313(1966).

70. C.D. Boley et al., Phys. Rev. 167, 1275(1968).

71. H. Gittleson et al., Phys. Rev. D (to be published).

72. C. Bernadini et al., Lettere Nuovo Cimento 1 , 15 (1969).

73. C.A. Lichtenstein et al., Phys. Kev. D1, 825(1970).

74. J.F. Crawford et al., Proposal No. 164 to the National Accelerator Laboratory (1971), unpublished.

75. D.B. Cline, Proceedings of the TVth International Conference on Neutrino Physics and Astrophystrs Philadelphia, 1974, to be published.

76. B. C. Barish et al., Phys. Rev. Letters 32, 1387(1974).

77. A. Rich and J.C. Wesley, Rev. Mod. Phys. 44, 250(1972).

78. C.S. Wu and L. Wilets, Ann. Rev. Sci. 12, 527(1969).

79. J. Bernstein, M. Rudermian and G. Feinberg, Phys. Rev. 132, 1227(1963).
80. A. DeRujula and B. Lautrup, Nuovo Cimento 3, 49(1972).

81. G. Backenstoss et aI., Phys. Letters 31B, 233(1970); M.S. Dixit et al., Physs. Rev. Letters 2], 878(1971); H.K. Walter et al., Phys. Letters 40B, 197 (1972).

82. Recent references and G.A. Rinker, Ir. and L. Wilets, Phys. Rev. Letters 31 , I559(1973); J. Arafune, Phys. Rev. Letters 32, 560(1974); L.s. Brown et al., Phys. Rev. Letters 22, 562(1974).

83. I. B. Okun and V.I. Zakharov, Nuclear Phys. B57, 252(1973).

84. For recent summaries see Proceedings of the 17th International Conference on High Energy Physics, 1974 (to be published).

85. M.J. Tannenbaum, paper presented to the Muon Physics Conference, Colorado State University, 1971.

86. J. J. Russell et $\underline{\text { al. }}$, Phys. Rev. Letters 26,46 (1971).

87. J.G. Rutherglen in Proceedings of the 4th International Symposium on Electron and Photon Interactions at High Energy. (Daresbury Nuclear Physics Laboratory, Daresbury, England, 1969).

88. R. Hofstadtex, Ann. Rev. Nucl. Sci. I, (1957).

89. M. L. Perl, High Energy Hadron Physics (Wiley, New York, 1974), Chapters 19 and 20.

90. K.J. Barnes, Nuovo Clmento 27, 228(1963).

91. R.W. Ellsworth et al., Phys. Rev. 165, 1449(1968).

92. L. Camilleri et al., Phys. Rev. Letters 23, 153(1969); L. Camilleri, Columbia University Nevis Laboratories Report No. 176.

93. I. Kostoulas et al., Phys. Rev. Letters 22, 489(1974).

94. A.B. Fntenberg, Ph.D. Thesis, Univ. of Rochester Report No. UR-476 (1974), unpublis hed.

95. T.J. Braunstein et 료., Phys. Rev. D6, 106(1972). 
96. M.I. Perl, Physics Today, July, 34(1971).

97. A. Entenberg et al., Phys. Rev. Letters 32, 486(1974).

98. I.J. Kim et al., Phys. Rev. Ietters 33, 551(1974).

99. D.J. Fox et al., Phys. Rev. Ietters (to be published).

100. W. C. Barber et 브., Phys. Rev. D3, 2796(1971).

101. H. Newman et ⒈ , Phys. Rev. Ietters 32, 483(1974); V. Silvestrini, Proccedings of the 16th International Conference on High Energy Physics (National Accelerator Laboratory, Batavia, 1973).

102. B. L. Beron et al., Phys. Rev. Letters 33, 663(1974).

103. A.M. Boyarski, Bull. Am. Phys. Soc. 19, 542(1973); B. Richter, Proceedings of the 17th International Conference on High Energy Physics (London, 1974).

104. B. Borgia et al., Lettere Nuovo Cimento 3, 115(1972); V. Alles-Borelli et al., Muovo Csmento 7 A, $330(1972)$.

105. F.J.M. Farley, unpublished paper entitled, The Status of Quantum Electrodynamics (Royal Military College of Science, Shrivenham, Swindon, England, 1969).

106. For the application of this equation to muon-proton elastic scattering see D. Kiang and S.H. Ng, Phys. Rev. D2, 1964(1970) and the references contained in that paper.

107. L. Yu. Kobzarev and L. B. Okun', Soviet Physics JETP 14, 859(1962); J. Expt1. Theoret. Phys. (U.S.S.R.) 41, 1205 (1961).

108. B. Richter, Invited talk at the Irvine Conference, December 1973 (unpublished); I.I.Yi Bigi and and J.D. Bjorken, Phys. Rev. (to be published); M.A.B. Beg and G. Feinberg, Phys. Rev. Letters 33, 606(197/); J.C. Pate and A. Salan, Phys. Rev. Letters 32, 1083(1974). 